\title{
Plantas brasileiras com ação anticolinesterásica - uma revisão
}

\author{
Brazilian plants with anticholinesterase action - a review \\ Plantas brasileñas con acción anticolinesterasa - una revisión
}

Recebido: 02/12/2021 | Revisado: 09/12/2021 | Aceito: 25/12/2021 | Publicado: 02/01/2022

Francisco Flávio da Silva Lopes

ORCID: https://orcid.org/0000-0003-2754-5521 Universidade Estadual do Ceará, Brasil E-mail: flaviollopez@gmail.com Lucas Soares Frota

ORCID: https://orcid.org/0000-0001-5583-5193 Universidade Estadual do Ceará, Brasil E-mail: lucassfrota@gmail.com Gilson Araújo Fontenele ORCID: https://orcid.org/0000-0002-5940-6983 Universidade Estadual do Ceará, Brasil E-mail: gilson_0410@hotmail.com

Marcus Vinícius Ferreira da Silva ORCID: https://orcid.org/0000-0001-6753-4371 Universidade Estadual do Ceará, Brasil E-mail: marcus.silva@aluno.uece.br

Victor Borges Fernandes

ORCID: https://orcid.org/0000-0003-4666-1026 Universidade Estadual do Ceará, Brasil E-mail: victor.fernandes@ aluno.uece.br

Renato Almeida Montes

ORCID: https://orcid.org/0000-0002-8644-8644 Universidade Estadual do Ceará, Brasil E-mail: renato.montes@aluno.uece.br

Selene Maia de Morais

ORCID: https://orcid.org/0000-0002-2766-3790 Universidade Estadual do Ceará, Brasil E-mail: selenemaiademorais@gmail.com

\begin{abstract}
Resumo
O Brasil possui uma grande flora distribuída por todo seu território, contendo a maior diversidade genética em espécies do mundo, muitas delas utilizadas para fins terapêutico. As plantas são as melhores fontes para a descoberta de compostos bioativos contra as doenças. A Doença de Alzheimer é uma doença neurodegenerativa caracterizada pela perda de memória, afeta principalmente a população com mais de 65 anos de idade. Este trabalho descreve um levantamento sobre extratos e óleos obtidos a partir de plantas da flora brasileira com potencial ação anticolinesterásica. Realizou-se uma revisão bibliográfica sistemática da produção científica em bases de dados da CAPES, Google Scholar, PubMed, Scielo, Science Direct, SciFinder, Scopus e Web of Science. Selecionou-se 41 artigos publicados entre 2011 e 2021. Foram identificadas 152 espécies vegetais distribuídas em 49 famílias botânicas, onde a Fabaceae foi a mais citada com 32 espécies $(21,05 \%)$. A produção de extrato foi a principal forma escolhida, 253 vezes $(93,36 \%)$. As folhas foram as partes mais utilizada, 94 vezes $(43,12 \%)$. As extrações por etanol se destacaram com 140 vezes $(51,66 \%)$. A maioria dos ensaios avaliou a inibição da acetilcolinesterase em microplaca de 96 poços, 182 ensaios (54,17\%). De todas as espécies relatadas 110 promoveram a inibição, desse total 66,36\% obtiveram uma alta intensidade. O estudo atingiu seu objetivo ao mostrar resultados relevantes na inibição da AChE, porém vale ressaltar que diante do tamanho da nossa flora, poucas espécies foram investigadas, tampouco foram testadas in vivo para se corroborar com os resultados dos ensaios in vitro.
\end{abstract}

Palavras-chave: Plantas brasileiras; Atividade anticolinesterásica; Doença de Alzheimer.

\begin{abstract}
Brazil has a large flora distributed throughout its territory, containing the greatest genetic diversity in species in the world, many of them used for therapeutic purposes. Plants are the best sources for discovering bioactive compounds against diseases. Alzheimer's Disease is a neurodegenerative disease characterized by memory loss, mainly affecting the population over 65 years of age. This work describes a survey of extracts and oils obtained from plants of the Brazilian flora with potential anticholinesterase action. A systematic literature review of scientific production in databases from CAPES, Google Scholar, PubMed, Scielo, Science Direct, SciFinder, Scopus and Web of Science was carried out. 41 articles published between 2011 and 2021 were selected. 152 plant species were identified, distributed
\end{abstract}


in 49 botanical families, where the Fabaceae was the most cited with 32 species $(21.05 \%)$. Extract production was the main form chosen, 253 times (93.36\%). The leaves were the most used parts, 94 times (43.12\%). Ethanol extractions stood out 140 times $(51.66 \%)$. Most assays evaluated acetylcholinesterase inhibition in 96-well microplate, 182 assays $(54.17 \%)$. Of all reported species, 110 promoted inhibitions, of which $66.36 \%$ had a high intensity. The study achieved its objective by showing relevant results in the inhibition of AChE, however it is worth mentioning that given the size of our flora, few species were investigated, nor were they tested in vivo to corroborate the results of the in vitro assays.

Keywords: Brazilian plants; Anticholinesterase activity; Alzheimer's disease.

\section{Resumen}

Brasil tiene una gran flora distribuida por todo su territorio, que contiene la mayor diversidad genética de especies del mundo, muchas de ellas utilizadas con fines terapéuticos. Las plantas son la mejor fuente para descubrir compuestos bioactivos contra las enfermedades. La enfermedad de Alzheimer es una enfermedad neurodegenerativa caracterizada por la pérdida de memoria, que afecta principalmente a la población mayor de 65 años. Este trabajo describe un relevamiento de extractos y aceites obtenidos de plantas de la flora brasileña con potencial acción anticolinesterasa. Se realizó una revisión bibliográfica sistemática de la producción científica en bases de datos de CAPES, Google Scholar, PubMed, Scielo, Science Direct, SciFinder, Scopus y Web of Science. Se seleccionaron cuarenta artículos publicados entre 2011 y 2021. Se identificaron 152 especies vegetales, distribuidas en 49 familias botánicas, donde la Fabaceae fue la más citada con 32 especies $(21,05 \%)$. La producción de extracto fue la forma principal elegida, 253 veces $(93,36 \%)$. Las hojas fueron las partes más utilizadas, 94 veces $(43,12 \%)$. Las extracciones con etanol se destacaron 140 veces $(51,66 \%)$. La mayoría de los ensayos evaluaron la inhibición de la acetilcolinesterasa en microplacas de 96 pocillos, 182 ensayos (54,17\%). De todas las especies notificadas, 110 promovieron la inhibición, de las cuales el 66,36\% tuvo una intensidad alta. El estudio alcanzó su objetivo al mostrar resultados relevantes en la inhibición de la AChE, sin embargo cabe mencionar que dado el tamaño de nuestra flora, pocas especies fueron investigadas, ni tampoco testadas in vivo para corroborar los resultados de los ensayos in vitro.

Palabras clave: Plantas brasileñas; Actividad anticolinesterasa; Enfermedad de Alzheimer.

\section{Introdução}

A utilização de produtos naturais para o tratamento de enfermidades é tão antiga quanto a própria humanidade, considerado uma prática milenar, está presente em várias culturas pelo mundo. Com o passar dos anos o conhecimento foi se atualizando e incorporando novos usos e práticas. Algumas comunidades tradicionais possui um amplo conhecimento etnobotânico, usando espécies vegetais como matéria-prima para combater muitas das doenças de forma segura e sustentável, em equilíbrio com a conservação do meio ambiente (Magalhães et al., 2022).

Os produtos naturais em especial, as plantas medicinais, são relativamente seguras em comparação com os fármacos sintéticas existentes, segundo a Organização Mundial da Saúde (OMS) 80\% das pessoas do planeta dependem de drogas fitoterápicas para combater as doenças (Patel et al., 2018).

O Brasil possui uma grande flora distribuída em todo seu território, contendo uma grande variedade de espécies de plantas, sendo a maior diversidade genética em espécies do mundo, muitas delas utilizadas para fins terapêutico pela população local, porém apenas cerca de $10 \%$ foram avaliadas quanto as suas características biológicas, e menos de 5\% foram submetidas a estudos fitoquímicos detalhado (Morais et al., 2020; Penido et al., 2016).

Com base no conhecimento empírico da população sobre as propriedades medicinais das plantas, os estudiosos em produtos naturais estão guiando suas investigações científicas e proporcionando grandes avanços na fitoquímica moderna e na química medicinal (Valli et al., 2016).

Morais et al. (2020) descreve em seu trabalho que os produtos naturais, as plantas, são a melhor fonte para se descobrir novos compostos bioativos. Entre as atuais estratégias utilizadas para a identificação e isolamento de compostos com potencial atividade biológica, os estudos com extratos de plantas se destacam como uma das mais importantes e significantes na busca por essas substâncias.

As espécies vegetais nos últimos anos proporcionaram diretamente ou indiretamente o desenvolvimento de cerca de dois terços dos novos medicamentos aprovados no mundo, muitos deles são utilizados para tratar distúrbios neurológicos 
associados à regulação da enzima AChE (Acetilcolinesterase) como a Doença de Alzheimer (Barbosa et al., 2020).

A Doença de Alzheimer (DA) é uma doença neurodegenerativa complexa, progressiva e multifatorial, caracterizada pela perda de memória e declínio cognitivo que pode afetar a fala, orientação visual espacial, comportamento e o sistema motor. Ela afeta principalmente a população idosa com mais de 65 anos de idade (10\% para pessoas com mais de 65 anos e 40\% para aqueles com mais de 80 anos), estima-se que mais de 47 milhões de indivíduos desenvolveram a doença em todo o mundo. Prevê-se que mais de 131 milhões de pessoas serão afetadas pela DA até 2050 (Deture \& Dickson, 2019).

Descrito pela primeira vez em 1906 pelo médico alemão Alois Alzheimer, a DA é considerado um dos maiores problemas de saúde pública do planeta, um dos principais problemas neurodegenerativo, podendo ser teoricamente revertido e/ou atenuado pela elevação dos níveis do neurotransmissor acetilcolina (ACh) na área sináptica neuronal (Silva et al., 2019). ACh é responsável pela mensagem de um neurônio a outro, age nas sinapses colinérgicas presentes no sistema nervoso central (SNC) e sistema nervoso periférico (SNP), sendo hidrolisada pela enzima AChE (Araújo et al., 2016).

A AChE é a serina hidrolase incumbida de finalizar a transmissão do impulso nervoso, degradando a $\mathrm{ACh}$ em acetato e colina. Os inibidores da acetilcolinesterase (IAChE) impedem esse processo, gerando um aumento no nível da ACh e consequentemente a redução dos sintomas cognitivos e funcionais (Barbosa et al., 2020; Oliveira et al., 2021).

O alcalóide fisostigmina foi o primeiro IAChE descoberto, em 1923 foi sintetizado a partir da Physostigma venenosum L., e desde então é um dos inibidores naturais mais utilizados em pesquisas científicas contra as doenças degenerativas (Araújo et al., 2016).

Os produtos naturais, em especial as espécies vegetais como as plantas superiores, são os principais alvos das atuais pesquisas com atividade inibitória da $\mathrm{AChE}$, sempre objetivando a descoberta por novas substâncias bioativas, que possuem menor valor e menos efeitos adversos em comparação as drogas sintéticas (Penido et al., 2017). Deste modo, o objetivo desta revisão foi buscar artigos científicos que focaram nas plantas da flora brasileira que demostraram potencial terapêutico contra a Doença de Alzheimer.

\section{Metodologia}

Este trabalho descreve um levantamento de literatura sobre extratos e óleos vegetais obtidos a partir de plantas da flora brasileira com potencial ação anticolinesterásica, para tanto foi realizado uma revisão bibliográfica sistemática seguindo os critérios propostos pela metodologia PRISMA (Principais Itens para Relatar Revisões Sistemáticas e Meta-Análises) (Galvão et al., 2015). Como estratégia de pesquisa utilizou-se a produção científica existente em bases de dados on-line de periódicos da CAPES, Google Scholar, PubMed, Scielo, Science Direct, SciFinder, Scopus e Web of Science, utilizando nas buscas as expressões: plantas brasileira, extratos vegetais, óleos vegetais e ação anticolinesterásica como palavras-chaves.

Como critérios de inclusão definiu-se a produção de artigos originais com livre acesso, desenvolvidos no Brasil com um intervalo de tempo de 10 anos para publicações compreendidas entre 2011 a 2021 nos idiomas português e inglês, também foram consultados capítulos de livros para seres usados com dados complementares. Os critérios de exclusão consistiram em artigos de revisões (sistemática, integrativa e narrativa), artigos de opinião, artigos sobre espécies que não eram de origem brasileira, monografias, dissertações e teses.

Ao final de todo processo, 41 estudos foram selecionados e incorporados à revisão sistemática por apresentarem relevantes informações sobre as espécies brasileiras (Figura 1). 
Figura 1. Fluxograma de seleção dos estudos.

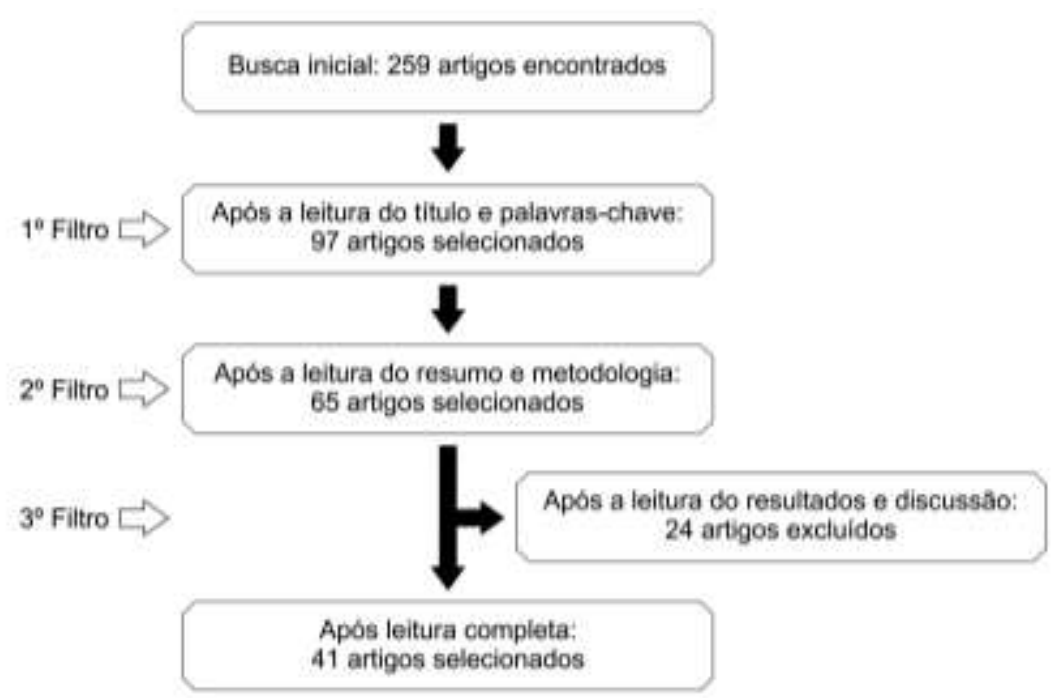

Fonte: Autores.

\section{Resultados e Discussão}

Após análise dos artigos selecionados, observou-se que nos últimos anos estão sendo realizados vários estudos científicos com espécies vegetais da flora nativa do Brasil, sempre objetivando a busca por novos fármacos ou compostos bioativos para combater a Doença de Alzheimer.

Santos et al. (2018), determinaram atividade anticolinesterase de extratos e frações de 54 plantas e classificaram a ação de acordo com a concentração média inibitória $\mathrm{CI}_{50}$ como: potência alta $\left(\mathrm{CI}_{50}<20 \mu \mathrm{g} \cdot \mathrm{mL}^{-1}\right)$; potência moderada $\left(20<\mathrm{CI}_{50}<\right.$ $\left.200 \mu \mathrm{g} \cdot \mathrm{mL}^{-1}\right)$ e potência baixa $\left(200<\mathrm{CI}_{50}<1000 \mu \mathrm{g} \cdot \mathrm{mL}^{-1}\right)$. Já Vinutha et al. (2007), classificou a intensidade da inibição como: potência alta $(>50 \%)$, potência moderada $(30-50 \%)$ e potência baixa $(<30 \%)$. Para a classificação das inibições promovidas nos ensaios com Cromatografia em Camada Delgada (CCD) utilizou-se como paramento o valor médio do halo formado pelo padrão fisostigmina: potência alta $(>0,7 \mathrm{~cm})$, potência moderada $(0,7-0,4 \mathrm{~cm})$ e potência baixa $(<0,4 \mathrm{~cm})$.

Desta forma pela revisão efetuada pode-se destacar algumas plantas como mais eficazes na inibição da AChE:

Barbosa et al. (2020), encontrou para o óleo essencial extraído das cascas de Myrciaria floribunda uma inibição da AChE com $\mathrm{CI}_{50}=0,08 \mu \mathrm{g} \cdot \mathrm{mL}^{-1}$. No estudo de Feitosa et al. (2011), Jatropha gossypiifolia apresentou $\mathrm{CI}_{50}=0,05 \mu \mathrm{g} \cdot \mathrm{mL}^{-1}$, Senna alata uma $\mathrm{CI}_{50}=0,08 \mu \mathrm{g} \cdot \mathrm{mL}^{-1}$, Ipomoea asarifolia com $\mathrm{CI}_{50}=0,12 \mu \mathrm{g} \cdot \mathrm{mL}^{-1}$, Kalanchoe brasiliensis com $\mathrm{CI}_{50}=0,16$ $\mu \mathrm{g} \cdot \mathrm{mL}^{-1}$ e Jatropha curcas com $\mathrm{CI}_{50}=0,25 \mu \mathrm{g} \cdot \mathrm{mL}^{-1}$. A pesquisa de Feitosa et al. (2017) revelou para o óleo essencial da Eugenia dysenterica uma $\mathrm{CI}_{50}=0,92 \mu \mathrm{g} \cdot \mathrm{mL}^{-1}$. Trabalho desenvolvido por Santos et al. (2012), mostrou para o extrato aquoso das folhas Erythrina velutina uma $\mathrm{CI}_{50}=0,57 \mathrm{mg} \cdot \mathrm{mL}^{-1}$.

Na Tabela 1 estão denominados as famílias, os nomes científicos, nomes populares, partes utilizadas, meios de extração, material produzido, ensaios utilizados, inibição e potência promovida pelas espécies vegetais utilizadas nos estudos para avaliar a atividade inibitória da enzima AChE. 
Research, Society and Development, v. 11, n. 1, e6211124262, 2022

(CC BY 4.0) | ISSN 2525-3409 | DOI: http://dx.doi.org/10.33448/rsd-v11i1.24262

\begin{tabular}{|c|c|c|c|c|c|c|c|c|c|}
\hline Família & Nome científico & Nome popular & Parte Utilizada & Meio para extração & $\begin{array}{c}\text { Amostra } \\
\text { produzida }\end{array}$ & Ensaio realizado & $\begin{array}{l}\text { Inibição } \\
\text { da AChE }\end{array}$ & Potência & Referências \\
\hline Amaranthaceae & Alternanthera brasiliana & Anador & Partes aéreas & $\mathrm{EtOH}$ & Extrato & $\mathrm{CCD}$ & Negativa & Nenhuma & Morais et al. (2013) \\
\hline Amaryllidaceae & Hippeastrum psittacinum & Açucena & Bulbos & $\mathrm{EtOH}$ & Extrato & Microdiluição & Positiva & Alta & Gasca et al. (2020) \\
\hline \multirow[t]{8}{*}{ Anacardiaceae } & Anacardium occidentale & Cajueiro-da-Praia & Casca do caule & $\mathrm{EtOH}$ & Extrato & Microdiluição & Positiva & Alta & Morais et al. (2021) \\
\hline & Mangifera indica & Mangueira & Folhas & EtOH e EtOH 70\% & Extrato & Microdiluição & Positiva & Alta & $\begin{array}{l}\text { Morais et al. (2021); } \\
\text { Penido et al. (2017) }\end{array}$ \\
\hline & Myracrodruon urundeuva & Aroeira-do-Sertão & $\begin{array}{l}\text { Casca do caule e } \\
\text { sementes }\end{array}$ & EtOH e EtOH 70\% & Extrato & Microdiluição & Positiva & Alta & $\begin{array}{l}\text { Morais et al. (2021); } \\
\text { Penido et al. (2017); } \\
\text { Farias et al. (2013) }\end{array}$ \\
\hline & Schinopsis brasiliensis & Baraúna & Sementes & $\mathrm{EtOH}$ & Extrato & Microdiluição & Negativa & Nenhuma & Farias et al. (2013) \\
\hline & Spondias mombin & Cajazeira & Folhas e partes aéreas & $\mathrm{EtOH}$ & Extrato & CCD e Microdiluição & Positiva & Moderada & $\begin{array}{l}\text { Morais et al. (2021); } \\
\text { Morais et al. (2013) }\end{array}$ \\
\hline & Spondia purpurea & Siriguela & $\begin{array}{l}\text { Sementes, pele e polpa } \\
\text { do fruto }\end{array}$ & $\mathrm{EtOH}$ & Extrato & $\mathrm{CCD}$ & Positiva & Alta & Omena et al. (2012) \\
\hline & Spondia tuberosa & Umbu & $\begin{array}{l}\text { Sementes, pele e polpa } \\
\text { do fruto }\end{array}$ & $\mathrm{EtOH}$ & Extrato & $\mathrm{CCD}$ & Positiva & Moderada & Omena et al. (2012) \\
\hline & Tapirira guianensis & Pau-Pombo & Folhas & $\mathrm{EtOH}$ & Extrato & Microdiluição & Positiva & Moderada & Morais et al. (2021) \\
\hline \multirow[t]{6}{*}{ Annonaceae } & Annona cacans & Araticum-Cagão & Folhas & MetOH & Extrato & CCD e Microdiluição & Negativa & Nenhuma & Formagio et al. (2015) \\
\hline & Annona coriacea & Fruta-do-Conde & $\begin{array}{l}\text { Folhas, sementes e } \\
\text { capítulo floral }\end{array}$ & MetOH & Extrato & CCD e Microdiluição & Positiva & Alta & Formagio et al. (2015) \\
\hline & Annona crassiflora & Araticum & Folhas e sementes & MetOH & Extrato & CCD e Microdiluição & Positiva & Moderada & Formagio et al. (2015) \\
\hline & Annona glabra & Araticum-do-Brejo & $\begin{array}{l}\text { Folhas, cascas do } \\
\text { caule e sementes }\end{array}$ & $\begin{array}{c}\mathrm{C}_{6} \mathrm{H}_{6}, \mathrm{CHCl}_{3}, \mathrm{EtOAc} \\
\text { e EtOH }\end{array}$ & Extrato & CCD e Microdiluição & Positiva & Alta & $\begin{array}{l}\text { Morais et al. (2021); } \\
\text { Silva et al. (2015) }\end{array}$ \\
\hline & Annona sylvatica & Pinha & Folhas e sementes & MetOH & Extrato & CCD e Microdiluição & Positiva & Baixa & Formagio et al. (2015) \\
\hline & Duguetia furfuracea & Ata-Brava & Folhas e sementes & $\mathrm{MetOH}$ & Extrato & CCD e Microdiluição & Positiva & Moderada & Formagio et al. (2015) \\
\hline
\end{tabular}


Research, Society and Development, v. 11, n. 1, e6211124262, 2022

(CC BY 4.0) | ISSN 2525-3409 | DOI: http://dx.doi.org/10.33448/rsd-v11i1.24262

\begin{tabular}{|c|c|c|c|c|c|c|c|c|c|}
\hline Apocynaceae & Hancornia speciosa & Mangaba & Cascas & EtOH $70 \%$ & Extrato & Microdiluição & Positiva & Alta & Penido et al. (2017) \\
\hline Arecaceae & Euterpe oleracea & Açaí & Semente & EtOH $70 \%$ & Extrato & Microdiluição & Positiva & Moderada & Penido et al. (2017) \\
\hline Asteraceae & Bellis perennis & Margarida & Flores & $\mathrm{EtOH}$ & Extrato & In vivo & Positiva & Alta & Marques et al. (2013) \\
\hline \multirow[t]{2}{*}{ Bignoniaceae } & Handroanthus impetiginosus & Ipê Roxo & Casca do caule & $\mathrm{EtOH}$ & Extrato & Microdiluição & Positiva & Alta & Morais et al. (2021) \\
\hline & Jacaranda oxyphylla & Caroba & Folhas e galhos & $\mathrm{C}_{6} \mathrm{H}_{6}, \mathrm{CHCl}_{3}$ e EtOH & Extrato & Microdiluição & Positiva & Alta & Pereira et al. (2015) \\
\hline Bixaceae & Cochlospermum regium & $\begin{array}{l}\text { Algodão-do- } \\
\text { Campo }\end{array}$ & Raiz & MetOH & Extrato & In vivo & Positiva & Alta & Pedroso et al. (2019) \\
\hline Boraginaceae & Cordia oncocalyx & $\begin{array}{l}\text { Pau-Branco-do- } \\
\text { Sertão }\end{array}$ & Casca do caule & $\mathrm{EtOH}$ & Extrato & Microdiluição & Positiva & Moderada & Morais et al. (2021) \\
\hline \multirow[t]{2}{*}{ Capparaceae } & Crateva tapia & Trapiá & Folhas & $\mathrm{EtOH}$ & Extrato & Microdiluição & Positiva & Alta & Morais et al. (2021) \\
\hline & Cynophalla flexuosa & Feijão-de-Boi & Vargens & $\mathrm{H}_{2} \mathrm{O}$ & Óleo essencial & Microdiluição & Positiva & Alta & Carvalho et al. (2021) \\
\hline Caryocaraceae & Caryocar coriaceum & Pequí & $\begin{array}{l}\text { Semente, pele e polpa } \\
\text { do fruto }\end{array}$ & $\mathrm{EtOH}$ & Extrato & $\mathrm{CCD}$ e microdiluição & Positiva & Alta & $\begin{array}{l}\text { Alves et al. (2017); } \\
\text { Farias et al. (2013) }\end{array}$ \\
\hline Celastraceae & Maytenus rigida & Bom-Nome & Casca do caule & $\mathrm{EtOH}$ e $\mathrm{H}_{2} \mathrm{O}$ & Extrato & Microdiluição & Positiva & Baixa & Mota et al. (2012) \\
\hline \multirow[t]{3}{*}{ Chrysobalanaceae } & Chrysobalanus icaco & Gragerú & Folhas & $\mathrm{EtOH}$ & Extrato & Microdiluição & Negativa & Nenhuma & Mota et al. (2012) \\
\hline & Licania rígida & Oiticica & Sementes & $\mathrm{EtOH}$ & Extrato & Microdiluição & Positiva & Alta & Farias et al. (2013) \\
\hline & Licania tomentosa & Oiti & Sementes & $\mathrm{EtOH}$ & Extrato & Microdiluição & Positiva & Baixa & Farias et al. (2013) \\
\hline \multirow[t]{2}{*}{ Combretaceae } & Laguncularia racemosa & Mangue-Branco & Folhas & $\mathrm{EtOH}$ & Extrato & Microdiluição & Positiva & Alta & Morais et al. (2021) \\
\hline & Terminalia catappa & Castanholeira & Folhas & EtOH & Extrato & Microdiluição & Positiva & Alta & Morais et al. (2021) \\
\hline Connaraceae & Connarus detersus & Cabelo-de-Negro & Sementes & $\mathrm{EtOH}$ & Extrato & Microdiluição & Positiva & Alta & Farias et al. (2013) \\
\hline \multirow[t]{2}{*}{ Convolvulaceae } & Ipomoea asarifolia & Salsa-Brava & Folhas e flores & EtOAc e $\mathrm{MeOH}$ & Extrato & CCD e Microdiluição & Positiva & Alta & Feitosa et al. (2011) \\
\hline & Ipomoea batatas & Batata-Doce & Folhas & EtOAc e $\mathrm{MeOH}$ & Extrato & CCD e Microdiluição & Positiva & Alta & Feitosa et al. (2011) \\
\hline \multirow[t]{2}{*}{ Crassulaceae } & Kalanchoe brasiliensis & Courama & Folhas & EtOAc e $\mathrm{MeOH}$ & Extrato & CCD e Microdiluição & Positiva & Alta & Feitosa et al. (2011) \\
\hline & Kalanchoe pinnata & Courama- & Folhas & EtOAc e $\mathrm{MeOH}$ & Extrato & CCD e Microdiluição & Positiva & Alta & Feitosa et al. (2011) \\
\hline
\end{tabular}


Research, Society and Development, v. 11, n. 1, e6211124262, 2022

(CC BY 4.0) | ISSN 2525-3409 | DOI: http://dx.doi.org/10.33448/rsd-v11i1.24262

\begin{tabular}{|c|c|c|c|c|c|c|c|c|c|}
\hline & & Vermelha & & & & & & & \\
\hline & Kalanchoe gastonis & $\begin{array}{l}\text { Courama- } \\
\text { Comprida }\end{array}$ & Folhas & EtOAc e $\mathrm{MeOH}$ & Extrato & CCD e Microdiluição & Positiva & Alta & Feitosa et al. (2011) \\
\hline \multirow[t]{7}{*}{ Euphorbiaceae } & Cnidoscolus quercifolius & Faveleira & $\begin{array}{l}\text { Folhas, raiz e casca da } \\
\text { raiz }\end{array}$ & MetOH & Extrato & $\mathrm{CCD}$ & Positiva & Alta & Paredes et al. (2016) \\
\hline & Croton blanchetianus & Marmeleiro-Preto & Folhas & $\mathrm{H}_{2} \mathrm{O}$ & Óleo essencial & Microdiluição & Positiva & Alta & Guerra et al. (2021) \\
\hline & Croton zehntneri & Canela-de-Cunhã & Partes aéreas & $\mathrm{EtOH}$ & Extrato & $\mathrm{CCD}$ & Positiva & Moderada & Morais et al. (2013) \\
\hline & Jatropha curcas & Pinhão-Manso & $\begin{array}{c}\text { Folhas e casca do } \\
\quad \text { caule }\end{array}$ & EtOAc e $\mathrm{MeOH}$ & Extrato & CCD e Microdiluição & Positiva & Alta & Feitosa et al. (2011) \\
\hline & Jatropha gossypifolia & Pinhão-Roxo & Folhas, caule e Látex & $\begin{array}{l}\text { EtOAc, } \mathrm{MeOH}, \\
\text { EtOH e } \mathrm{H}_{2} \mathrm{O}\end{array}$ & Extratos & CCD e Microdiluição & Positiva & Alta & $\begin{array}{l}\text { Martins et al. (2018); } \\
\text { Feitosa et al. (2011) }\end{array}$ \\
\hline & Jatropha pohliana & Pião-Bravo & Folhas & EtOAc e $\mathrm{MeOH}$ & Extrato & CCD e Microdiluição & Positiva & Alta & Feitosa et al. (2011) \\
\hline & Phyllanthus amarus & Quebra-Pedra & Folhas e flores & EtOAc e $\mathrm{MeOH}$ & Extrato & CCD e Microdiluição & Positiva & Alta & Feitosa et al. (2011) \\
\hline \multirow[t]{11}{*}{ Fabaceae } & Amburana cearensis & Cumarú & Sementes & $\mathrm{EtOH}$ & Extrato & Microdiluição & Negativa & Nenhuma & Farias et al. (2013) \\
\hline & Anadenanthera macrocarpa & Angico-Vermelho & Sementes & $\mathrm{EtOH}$ & Extrato & Microdiluição & Positiva & Alta & Farias et al. (2013) \\
\hline & Anadenanthera pavonina & Falso-Sândalo & Sementes & EtOH & Extrato & Microdiluição & Negativa & Nenhuma & Farias et al. (2013) \\
\hline & Anadenanthera peregrina & Angico & Cascas & EtOH $70 \%$ & Extrato & Microdiluição & Positiva & Baixa & Penido et al. (2017) \\
\hline & Bauhinia cheilantha & Mororó-do-Sertão & Cascas do caule & $\mathrm{EtOH}$ & Extrato & Microdiluição & Negativa & Nenhuma & Mota et al. (2012) \\
\hline & Bauhinia forficata & Mororó & Folhas & EtOH e EtOH 70\% & Extrato & Microdiluição & Positiva & Alta & $\begin{array}{l}\text { Morais et al. (2021); } \\
\text { Penido et al. (2017) }\end{array}$ \\
\hline & Bauhinia monandra & Pata-de-Vaca & Folhas, flores e galhos & $\mathrm{EtOH}$ & Extrato & Microdiluição & Positiva & Moderada & Oliveira et al. (2021) \\
\hline & Caesalpinia pulcherrima & Flamboyant mirim & Folhas & $\mathrm{EtOH}$ & Extrato & Microdiluição & Positiva & Alta & Morais et al. (2021) \\
\hline & Cassia alata & Canafístula & Partes aéreas & $\mathrm{EtOH}$ & Extrato & $\mathrm{CCD}$ & Negativa & Nenhuma & Morais et al. (2013) \\
\hline & Cassia fistula & Chuva-de-Ouro & Folhas e flores & EtOAc e MeOH & Extrato & CCD e Microdiluição & Positiva & Alta & Feitosa et al. (2011) \\
\hline & Cenostigma pyramidale & Catingueira & Folhas & EtOH & Extrato & Microdiluição & Positiva & Alta & Morais et al. (2021) \\
\hline
\end{tabular}


Research, Society and Development, v. 11, n. 1, e6211124262, 2022

(CC BY 4.0) | ISSN 2525-3409 | DOI: http://dx.doi.org/10.33448/rsd-v11i1.24262

\begin{tabular}{|c|c|c|c|c|c|c|c|c|}
\hline Copaifera langsdorffii & Copaiba & Cascas & EtOH $70 \%$ & Extrato & Microdiluição & Positiva & Alta & Penido et al. (2017) \\
\hline Copaifera officinalis & Copaíba & Resina & $\mathrm{H}_{2} \mathrm{O}$ & Óleo essencial & Microdiluição & Positiva & Alta & Lima et al. (2020) \\
\hline Dioclea megacarpa & Mucuna & Sementes & $\mathrm{EtOH}$ & Extrato & Microdiluição & Negativa & Nenhuma & Farias et al. (2013) \\
\hline Enterolobium contortisiliquum & Orelha-de-Negro & Sementes & $\mathrm{EtOH}$ & Extrato & Microdiluição & Negativa & Nenhuma & Farias et al. (2013) \\
\hline Erythrina velutina & Mulungú & Folhas & $\mathrm{C}_{6} \mathrm{H}_{6}$, EtOH e $\mathrm{H}_{2} \mathrm{O}$ & Extrato & $\begin{array}{l}\text { Microdiluição e in } \\
\text { vivo }\end{array}$ & Positiva & Moderada & $\begin{array}{l}\text { Morais et al. (2021); } \\
\text { Santos et al. (2012); } \\
\text { Mota et al. (2012) }\end{array}$ \\
\hline Geoffroea spinosa & Marizeira & Folhas & $\mathrm{EtOH}$ & Extrato & Microdiluição & Positiva & Moderada & Morais et al. (2021) \\
\hline Hymenaea courbaril & Jatobá & Sementes & $\mathrm{EtOH}$ & Extrato & Microdiluição & Negativa & Nenhuma & Farias et al. (2013) \\
\hline Hymenaea stigonocarpa & Jatobá-do-Sertão & Casca & $\mathrm{EtOH}$ & Extrato & Microdiluição & Positiva & Moderada & Morais et al. (2021) \\
\hline Leucaena leucocephala & Leucena & Folhas & EtOAc e $\mathrm{MeOH}$ & Extrato & CCD e Microdiluição & Positiva & Alta & Feitosa et al. (2011) \\
\hline Libidibia ferrea & Jucá & Folhas, cascas e fruto & $\begin{array}{c}\text { EtOAc, } \mathrm{MeOH} \text { e } \\
\text { EtOH }\end{array}$ & Extrato & CCD e Microdiluição & Positiva & Alta & $\begin{array}{l}\text { Morais et al. (2021); } \\
\text { Feitosa et al. (2011) }\end{array}$ \\
\hline Lonchocarpus sericeus & Ingá & Sementes & $\mathrm{EtOH}$ & Extrato & Microdiluição & Negativa & Nenhuma & Farias et al. (2013) \\
\hline Luetzelburgia auriculata & Pau-Moco & Sementes & EtOH & Extrato & Microdiluição & Negativa & Nenhuma & Farias et al. (2013) \\
\hline Mimosa tenuiflora & Jurema Preta & Folhas & $\mathrm{EtOH}$ & Extrato & Microdiluição & Positiva & Moderada & Morais et al. (2021) \\
\hline Parkia platycephala & Faveira & Folhas e Sementes & $\begin{array}{c}\mathrm{C}_{6} \mathrm{H}_{6}, \mathrm{MetOH}, \mathrm{EtOH} \\
\text { e EtOH } 70 \%\end{array}$ & Extrato & Microdiluição & Positiva & Alta & $\begin{array}{l}\text { Fernandes et al. (2021); } \\
\text { Farias et al. (2013) }\end{array}$ \\
\hline Piptadenia moniliformis & Catanduva & Sementes & $\mathrm{EtOH}$ & Extrato & Microdiluição & Positiva & Alta & Farias et al. (2013) \\
\hline Plathymenia reticulata & Candeia & Folhas & EtOH $70 \%$ & Extrato & Microdiluição & Positiva & Moderada & Penido et al. (2017) \\
\hline Senna alata & Fedegoso-Gigante & Folhas & EtOAc e $\mathrm{MeOH}$ & Extrato & CCD e Microdiluição & Positiva & Alta & Feitosa et al. (2011) \\
\hline Senna obtusifolia & Mata-Pasto & Sementes & $\mathrm{EtOH}$ & Extrato & Microdiluição & Negativa & Nenhuma & Farias et al. (2013) \\
\hline Senna reticulata & Maria-Mole & Folhas & EtOAc e $\mathrm{MeOH}$ & Extrato & CCD e Microdiluição & Positiva & Alta & Feitosa et al. (2011) \\
\hline Stryphnodendron coriaceum & Barbatimão & Folhas & EtOH 70\% & Extrato & Microdiluição & Positiva & Alta & Penido et al. (2017) \\
\hline
\end{tabular}


Research, Society and Development, v. 11, n. 1, e6211124262, 2022

(CC BY 4.0) | ISSN 2525-3409 | DOI: http://dx.doi.org/10.33448/rsd-v11i1.24262

\begin{tabular}{|c|c|c|c|c|c|c|c|c|c|}
\hline Geraniaceae & Pelargonium $x$ ssp & Gerânio & Flores & $\mathrm{H}_{2} \mathrm{O}$ & Óleo essencial & Microdiluição & Positiva & Alta & Lima et al. (2020) \\
\hline \multirow[t]{5}{*}{ Lamiaceae } & Marsypianthes chamaedrys & Betônica-Brava & Partes aéreas & EtOH & Extrato & CCD & Negativa & Nenhuma & Morais et al. (2013) \\
\hline & Mentha $x$ villosa & Menta & Partes aéreas & $\mathrm{EtOH}$ & Extrato & $\mathrm{CCD}$ & Negativa & Nenhuma & Morais et al. (2013) \\
\hline & Ocimum gratissimum & Alfavaca & Partes aéreas & $\mathrm{EtOH}$ & Extrato & CCD & Positiva & Moderada & Morais et al. (2013) \\
\hline & Ocimum selloi & Alfavaca & Partes aéreas & $\mathrm{EtOH}$ & Extrato & $\mathrm{CCD}$ & Negativa & Nenhuma & Morais et al. (2013) \\
\hline & Vitex gardneriana & Jaramataia & Folhas & $\mathrm{H}_{2} \mathrm{O}$ & Óleo essencial & Microdiluição & Positiva & Alta & Pereira et al. (2018) \\
\hline \multirow[t]{13}{*}{ Lauraceae } & Aniba panurensis & Louro-Amarelo & Folhas e galhos & $\mathrm{EtOH}$ & Microdiluição & $\mathrm{CCD}$ & Positiva & $\begin{array}{c}\text { Não } \\
\text { mensurado }\end{array}$ & Yamaguchi et al. (2012) \\
\hline & Aniba rosaeodora & Pau-Rosa & Galhos & $\mathrm{EtOH}$ & Extrato & $\mathrm{CCD}$ & Negativo & Nenhuma & Yamaguchi et al. (2012) \\
\hline & Cinnamomum zeylanicum & $\begin{array}{l}\text { Caneleira- } \\
\text { Verdadeira }\end{array}$ & Folhas e sementes & $\mathrm{C}_{6} \mathrm{H}_{6}, \mathrm{MetOH}$ e $\mathrm{H}_{2} \mathrm{O}$ & $\begin{array}{l}\text { Extrato e óleo } \\
\text { essencial }\end{array}$ & Microdiluição & Positiva & Alta & $\begin{array}{l}\text { Ribeiro et al. (2019); } \\
\text { Dalai et al. (2014) }\end{array}$ \\
\hline & Dicypellium manausense & Louro-Preto & Folhas e galhos & $\mathrm{EtOH}$ & Extrato & $\mathrm{CCD}$ & Negativo & Nenhuma & Yamaguchi et al. (2012) \\
\hline & Endlicheria citriodora & Eucalipto-Limão & Folhas e galhos & $\mathrm{EtOH}$ & Extrato & $\mathrm{CCD}$ & Positiva & $\begin{array}{c}\text { Não } \\
\text { mensurado }\end{array}$ & Yamaguchi et al. (2012) \\
\hline & Endlicheria sericea & Louro & Folhas e galhos & $\mathrm{EtOH}$ & Extrato & $\mathrm{CCD}$ & Negativo & Nenhuma & Yamaguchi et al. (2012) \\
\hline & Licaria cannella & Louro-Pirarucú & Folhas e galhos & $\mathrm{EtOH}$ & Extrato & $\mathrm{CCD}$ & Positiva & $\begin{array}{c}\text { Não } \\
\text { mensurado }\end{array}$ & Yamaguchi et al. (2012) \\
\hline & Licaria martiniana & Louro-Chumbo & Folhas e galhos & $\mathrm{EtOH}$ & Extrato & $\mathrm{CCD}$ & Positiva & $\begin{array}{c}\text { Não } \\
\text { mensurado }\end{array}$ & Yamaguchi et al. (2012) \\
\hline & Mezilaurus duckei & $\begin{array}{l}\text { Louro-Itaúba- } \\
\text { Abacate }\end{array}$ & Folhas e galhos & $\mathrm{EtOH}$ & Extrato & $\mathrm{CCD}$ & Negativo & Nenhuma & Yamaguchi et al. (2012) \\
\hline & Mezilaurus itauba & Louro-Itaúba & Folhas & $\mathrm{EtOH}$ & Extrato & $\mathrm{CCD}$ & Negativo & Nenhuma & Yamaguchi et al. (2012) \\
\hline & Ocotea ceanothifolia & Canela-Loura & Folhas e galhos & $\mathrm{EtOH}$ & Extrato & $\mathrm{CCD}$ & Positiva & $\begin{array}{c}\text { Não } \\
\text { mensurado }\end{array}$ & Yamaguchi et al. (2012) \\
\hline & Ocotea leucoxylon & Louro & Folhas e galhos & $\mathrm{EtOH}$ & Extrato & $\mathrm{CCD}$ & Positiva & $\begin{array}{c}\text { Não } \\
\text { mensurado }\end{array}$ & Yamaguchi et al. (2012) \\
\hline & Ocotea minor & Louro-Preto & Folhas e galhos & EtOH & Extrato & $\mathrm{CCD}$ & Positiva & Não & Yamaguchi et al. (2012) \\
\hline
\end{tabular}


Research, Society and Development, v. 11, n. 1, e6211124262, 2022

(CC BY 4.0) | ISSN 2525-3409 | DOI: http://dx.doi.org/10.33448/rsd-v11i1.24262

\begin{tabular}{|c|c|c|c|c|c|c|c|c|c|}
\hline & & & & & & & & mensurado & \\
\hline & Ocotea nigrescens & Louro-Santo & Folhas & EtOH & Extrato & $\mathrm{CCD}$ & Negativa & Nenhuma & Yamaguchi et al. (2012) \\
\hline & Ocotea splendens & Cedro-Pardo & Folhas & $\mathrm{EtOH}$ & Extrato & $\mathrm{CCD}$ & Negativa & Nenhuma & Yamaguchi et al. (2012) \\
\hline & Pleurothyrium vasquezii & $\begin{array}{c}\text { Louro- } \\
\text { Abacaterana }\end{array}$ & Folhas & $\mathrm{EtOH}$ & Extrato & $\mathrm{CCD}$ & Negativa & Nenhuma & Yamaguchi et al. (2012) \\
\hline & $\begin{array}{l}\text { Rhodostemonodaphne } \\
\text { crenaticupula }\end{array}$ & Louro-Branco & Folhas e galhos & $\mathrm{EtOH}$ & Extrato & CCD & Negativa & Nenhuma & Yamaguchi et al. (2012) \\
\hline & $\begin{array}{c}\text { Rhodostemonodaphne } \\
\text { negrensis }\end{array}$ & Louro-Fofo & Folhas & $\mathrm{EtOH}$ & Extrato & $\mathrm{CCD}$ & Positiva & $\begin{array}{c}\text { Não } \\
\text { mensurado }\end{array}$ & Yamaguchi et al. (2012) \\
\hline & $\begin{array}{l}\text { Rhodostemonodaphne } \\
\text { parvifolia }\end{array}$ & Louro & Folhas & EtOH & Extrato & CCD & Positiva & $\begin{array}{c}\text { Não } \\
\text { mensurado }\end{array}$ & Yamaguchi et al. (2012) \\
\hline & Rhodostemonodaphne recurva & Louro & Folhas e galhos & EtOH & Extrato & $\mathrm{CCD}$ & Positiva & $\begin{array}{c}\text { Não } \\
\text { mensurado }\end{array}$ & Yamaguchi et al. (2012) \\
\hline & Sextonia rubra & Louro-Vermelho & Folhas e galhos & $\mathrm{EtOH}$ & Extrato & $\mathrm{CCD}$ & Negativa & Nenhuma & Yamaguchi et al. (2012) \\
\hline Lecythidaceae & Couroupita guianensis & Abricó de macaco & Folhas & $\mathrm{EtOH}$ & Extrato & Microdiluição & Positiva & Moderada & Morais et al. (2021) \\
\hline \multirow[t]{2}{*}{ Malpighiaceae } & Banisteriopsis anisandra & Liana & Folhas & $\begin{array}{c}\mathrm{C}_{6} \mathrm{H}_{6}, \mathrm{CHCl}_{3}, \mathrm{EtOAc} \\
\text { e } \mathrm{MeOH}\end{array}$ & Extrato & CCD & Positiva & $\begin{array}{c}\text { Não } \\
\text { mensurada }\end{array}$ & Frias et al. (2011) \\
\hline & Malpighia glabra & Acerola & Partes aéreas & $\mathrm{EtOH}$ & Extrato & $\mathrm{CCD}$ & Positiva & Moderada & Morais et al. (2013) \\
\hline \multirow[t]{4}{*}{ Malvaceae } & Ceiba pentandra & Paineira & Casca do caule & EtOH 70\% & Extrato & Microdiluição & Positiva & Alta & Morais et al. (2021) \\
\hline & Gossypium herbaceum & Algodoeiro & Folhas & EtOAc e $\mathrm{MeOH}$ & Extrato & CCD e Microdiluição & Positiva & Alta & Feitosa et al. (2011) \\
\hline & Guazuma ulmifolia & Mutamba & Folhas & $\mathrm{EtOH}$ & Extrato & CCD & Positiva & Alta & Morais et al. (2017) \\
\hline & Luehea divaricata & Açoita-Cavalo & Folhas & $\mathrm{EtOH}$ & Extrato & Microdiluição & Positiva & Moderada & Penido et al. (2017) \\
\hline Meliaceae & Trichilia catigua & Catuaba & Casca do caule & $\begin{array}{c}\mathrm{C}_{6} \mathrm{H}_{6}, \mathrm{CHCl}_{3}, \mathrm{EtOH} \\
70 \% \text { e } \mathrm{H}_{2} \mathrm{O}\end{array}$ & Extrato & Microdiluição & Positiva & Baixa & Martins et al. (2018) \\
\hline \multirow[t]{2}{*}{ Moraceae } & Brosimum gaudichaudii & Inharé & Folhas & $\mathrm{EtOH}$ & Extrato & Microdiluição & Positiva & Moderada & Morais et al. (2021) \\
\hline & Ficus benjamina & Berigan & Folhas & EtOAc e MeOH & Extrato & CCD e Microdiluição & Positiva & Alta & Feitosa et al. (2011) \\
\hline
\end{tabular}


Research, Society and Development, v. 11, n. 1, e6211124262, 2022

(CC BY 4.0) | ISSN 2525-3409 | DOI: http://dx.doi.org/10.33448/rsd-v11i1.24262

\begin{tabular}{|c|c|c|c|c|c|c|c|c|c|}
\hline Moringaceae & Moringa oleifera & Moringa & Folhas e sementes & $\mathrm{EtOH}$ & Extrato & Microdiluição & Positiva & Moderada & $\begin{array}{l}\text { Morais et al. (2021); } \\
\text { Mota et al. (2012) }\end{array}$ \\
\hline \multirow[t]{7}{*}{ Myrtaceae } & Eugenia dysenterica & Cagaita & Folhas & $\mathrm{H}_{2} \mathrm{O}$ & Óleo essencial & Microdiluição & Positiva & Alta & Feitosa et al. (2017) \\
\hline & Eugenia sulcata & Pitanga-Preta & Folhas & $\mathrm{H}_{2} \mathrm{O}$ & Óleo essencial & Microdiluição & Positiva & Alta & Lima et al. (2012) \\
\hline & Eugenia uniflora & Pitanga & Partes aéreas & EtOH & Extrato & CCD & Positiva & Moderada & Morais et al. (2013) \\
\hline & Myrciaria floribunda & Cambiuva & Pele do fruto & $\mathrm{H}_{2} \mathrm{O}$ & Óleo essencial & Microdiluição & Positiva & Alta & Barbosa et al. (2020) \\
\hline & Psidium guajava & Goiaba & Folhas & EtOH $70 \%$ & Extrato & Microdiluição & Positiva & Alta & Penido et al. (2017) \\
\hline & Syzygium aromaticum & Cravo-da-Índia & Fruto & EtOH $70 \%$ & Extrato & Microdiluição & Positiva & Moderada & Penido et al. (2017) \\
\hline & Syzygium cumini & Azeitona-Preta & Folhas & EtOH & Extrato & Microdiluição & Positiva & Moderada & Morais et al. (2021) \\
\hline Nyctaginaceae & Bougainvillea glabra & Três-Marias & Folhas e flores & EtOAc e $\mathrm{MeOH}$ & Extrato & CCD e Microdiluição & Positiva & Alta & Feitosa et al. (2011) \\
\hline Ochnaceae & Ouratea fieldingiana & Batiputá & $\begin{array}{l}\text { Folhas, galhos e } \\
\text { sementes }\end{array}$ & $\begin{array}{c}\mathrm{C}_{6} \mathrm{H}_{6}, \text { EtOH, } \mathrm{H}_{2} \mathrm{O} e \\
\text { Calor }\end{array}$ & $\begin{array}{l}\text { Extrato e óleo } \\
\quad \text { fixo }\end{array}$ & CCD e Microdiluição & Positiva & Alta & $\begin{array}{l}\text { Frota et al. (2021); } \\
\text { Nascimento et al. (2018) }\end{array}$ \\
\hline \multirow[t]{2}{*}{ Phyllanthaceae } & Phyllanthus amarus & Quebra-Pedra & Partes aéreas & EtOH & Extrato & $\mathrm{CCD}$ & Positiva & Moderada & Morais et al. (2013) \\
\hline & Plectranthus ornatus & Falso-Boldo & Partes aéreas & EtOH & Extrato & CCD & Positiva & Alta & Morais et al. (2013) \\
\hline \multirow[t]{3}{*}{ Piperaceae } & Piper aleyreanum & Piper & Partes aéreas & $\mathrm{H}_{2} \mathrm{O}$ & Óleo essencial & CCD & Positiva & Baixa & Silva et al. (2014) \\
\hline & Piper anonifolium & Piper & Partes aéreas & $\mathrm{H}_{2} \mathrm{O}$ & Óleo essencial & CCD & Positiva & Alta & Silva et al. (2014) \\
\hline & Piper hispidum & Piper & Partes aéreas & $\mathrm{H}_{2} \mathrm{O}$ & Óleo essencial & CCD & Positiva & Alta & Silva et al. (2014) \\
\hline \multirow[t]{2}{*}{ Poaceae } & Cymbopogon flexиosus & Capim-Limão & Folhas & $\mathrm{H}_{2} \mathrm{O}$ & Óleo essencial & Microdiluição & Positiva & Alta & Lima et al. (2020) \\
\hline & Digitaria insularis & Capim-Amargoso & Folhas & $\mathrm{C}_{6} \mathrm{H}_{6}$ e EtOAc & Extrato & Microdiluição & Positiva & Moderada & Santos et al. (2018) \\
\hline Polygalaceae & Bredemeyera floribunda & Pau-Caixão & Partes aéreas & EtOH & Extrato & CCD & Negativa & Nenhuma & Morais et al. (2013) \\
\hline Polygonaceae & Triplaris gardneriana & Pajeú & Sementes & EtOH & Extrato & Microdiluição & Positiva & Alta & Farias et al. (2013) \\
\hline Punicaceae & Punica granatum & Romã & Partes aéreas & EtOH & Extrato & CCD & Negativa & Nenhuma & Morais et al. (2013) \\
\hline Rhamnaceae & Ziziphus joazeiro & Juazeiro & Folhas e sementes & EtOH & Extrato & Microdiluição & Positiva & Alta & $\begin{array}{l}\text { Morais et al. (2021); } \\
\text { Farias et al. (2013) }\end{array}$ \\
\hline
\end{tabular}


Research, Society and Development, v. 11, n. 1, e6211124262, 2022

(CC BY 4.0) | ISSN 2525-3409 | DOI: http://dx.doi.org/10.33448/rsd-v11i1.24262

\begin{tabular}{|c|c|c|c|c|c|c|c|c|c|}
\hline Rubiaceae & Genipa americana & Jenipapo & $\begin{array}{l}\text { Folhas, sementes, pele } \\
\text { e polpa do fruto }\end{array}$ & EtOH & Extrato & CCD e Microdiluição & Positiva & Alta & $\begin{array}{l}\text { Morais et al. (2021); } \\
\text { Omena et al. (2012) }\end{array}$ \\
\hline & Tocoyena formosa & Jenipapo-Bravo & Folhas & EtOH & Extrato & Microdiluição & Positiva & Moderada & Morais et al. (2021) \\
\hline \multirow[t]{2}{*}{ Rutaceae } & Citrus limmonia & Cravo-Limão & Folhas & EtOAc e MeOH & Extrato & CCD e Microdiluição & Positiva & Alta & Feitosa et al. (2011) \\
\hline & Zanthoxylum syncarpum & Limãozinho & Folhas & EtOH & Extrato & CCD & Positiva & Moderada & Silva et al. (2014) \\
\hline Salicaceae & Prockia crucis & Cuiteiro & $\begin{array}{l}\text { Folhas e cascas do } \\
\text { caule }\end{array}$ & EtOH & Extrato & CCD & Positiva & Alta & Calixto Jr et al. (2015) \\
\hline \multirow[t]{2}{*}{ Sapindaceae } & Sapindus saponaria & Sabonete & Sementes & $\mathrm{EtOH}$ & Extrato & Microdiluição & Positiva & Alta & Farias et al. (2013) \\
\hline & Talisia esculenta & Pitombeira & Sementes & $\mathrm{EtOH}$ & Extrato & Microdiluição & Positiva & Alta & $\begin{array}{l}\text { Morais et al. (2021); } \\
\text { Dantas et al. (2020); } \\
\text { Farias et al. (2013) }\end{array}$ \\
\hline Sapotaceae & Sideroxylon obtusifolium & Quixabeira & Folhas & EtOH & Extrato & Microdiluição & Negativa & Nenhuma & Mota et al. (2012) \\
\hline Schrophulariaceae & Capraria biflora & Chá-de-Calçada & Partes aéreas & $\mathrm{EtOH}$ & Extrato & CCD & Negativa & Nenhuma & Morais et al. (2013) \\
\hline Sterculiceae & Guazuma ulmifolia & Mutamba & Folhas & EtOH & Extrato & Microdiluição & Positiva & Moderada & Morais et al. (2021) \\
\hline Urticaceae & Cecropia pachystachia & Torém & Folhas e partes aéreas & EtOH & Extrato & CCD e Microdiluição & Positiva & Alta & $\begin{array}{l}\text { Morais et al. (2021); } \\
\text { Morais et al. (2013) }\end{array}$ \\
\hline \multirow[t]{6}{*}{ Verbenaceae } & Hyptis fruticosa & $\begin{array}{l}\text { Alecrim-de- } \\
\text { Tabuleiro }\end{array}$ & Folhas & EtOH & Extrato & Microdiluição & Positiva & Baixa & Mota et al. (2012) \\
\hline & Lantana camara & Camará & Flores & $\mathrm{H}_{2} \mathrm{O}$ & Óleo essencial & Microdiluição & Positiva & Alta & Santos et al. (2015) \\
\hline & Lippia alba & Erva-Cidereira & Partes aéreas & EtOH & Extrato & CCD & Positiva & Alta & Morais et al. (2013) \\
\hline & Lippia hirta & Lippia & Folhas & EtOH & Extrato & Microdiluição & Positiva & Alta & Oliveira et al. (2019) \\
\hline & Lippia microphylla & $\begin{array}{l}\text { Alecrim-de- } \\
\text { Tabuleiro }\end{array}$ & Partes aéreas & EtOH & Extrato & CCD & Positiva & Moderada & Morais et al. (2013) \\
\hline & Vitex agnus-castus & Pimenta-da-Costa & Folhas & $\mathrm{EtOH}$ e $\mathrm{H}_{2} \mathrm{O}$ & Extrato & Microdiluição & Positiva & Alta & Mota et al. (2012) \\
\hline Viscaceae & Phoradendron piperoides & $\begin{array}{l}\text { Enxerto-de- } \\
\text { Passarinho }\end{array}$ & Folhas & $\mathrm{EtOH}$ e $\mathrm{H}_{2} \mathrm{O}$ & Extrato & Microdiluição & Positiva & Moderada & Mota et al. (2012) \\
\hline
\end{tabular}

Legenda: $\mathrm{C}_{6} \mathrm{H}_{6}$ (Hexano); $\mathrm{CHCl}_{3}$ (Clorofórmio), EtOAc (Acetato de Etila); $\mathrm{MeOH}$ (Metanol); EtOH (Etanol); $\mathrm{H}_{2} \mathrm{O}$ (Água).

Fonte: Autores. 


\section{Estudos da atividade anticolinesterásica com avaliação da concentração mínima inibitória}

No estudo de Feitosa et al. (2011), com extratos metanólicos e de acetato de etila de folhas, flores e casca de 18 espécies de plantas das famílias Convolvulaceae, Crassulaceae, Euphorbiaceae, Leguminosae, Malvaceae, Moraceae, Nyctaginaceae e Rutaceae, indicou que os extratos com melhores resultados no ensaio de diluição em microplaca de 96 poços pelo método de Ellman et al. (1961), modificado por Rhee et al. (2001), foram os foliares de Pinhão-Roxo (Jatropha gossypiifolia - Euphorbiaceae) com concentração inibitória para 50\% da $\mathrm{AChE}\left(\mathrm{CI}_{50}\right)$ igual 0,05 $\mu \mathrm{g} \cdot \mathrm{mL}^{-1}$, Fedegoso-Gigante (Senna alata) com $\mathrm{CI}_{50}=0,08 \mu \mathrm{g} \cdot \mathrm{mL}^{-1}$, Salsa-Brava (Ipomoea asarifolia - Convolvulaceae) com $\mathrm{CI}_{50}=0,12 \mu \mathrm{g} \cdot \mathrm{mL}^{-1}$, Courama (Kalanchoe brasiliensis - Crassulaceae) com $\mathrm{CI}_{50}=0,16 \mu \mathrm{g} \cdot \mathrm{mL}^{-1} \mathrm{e}$ Pinhão-Manso (Jatropha curcas - Euphorbiaceae) com $\mathrm{CI}_{50}=0,25 \mu \mathrm{g} \cdot \mathrm{mL}^{-1}$.

O fracionamento bioguiado dos extratos mais ativos de Kalanchoe brasiliensis resultou no isolamento de uma mistura ativa de três flavonóides: 8-methoxiquercetina, 8-methoxiquercetina-3,7-di- $O$-ramnopiranoside e 8-methoxicanferol-3,7-di- $O$ ramnopiranoside. No teste da cromatografia em camada delgada (CCD), esses flavonóides mostraram um efeito inibidor da acetilcolinesterase (Trevisan et al, 2006). A Figura 2 apresenta a estrutura dos compostos isolados.

Figura 2. Flavonoides ativos isolados da K. brasiliensis.

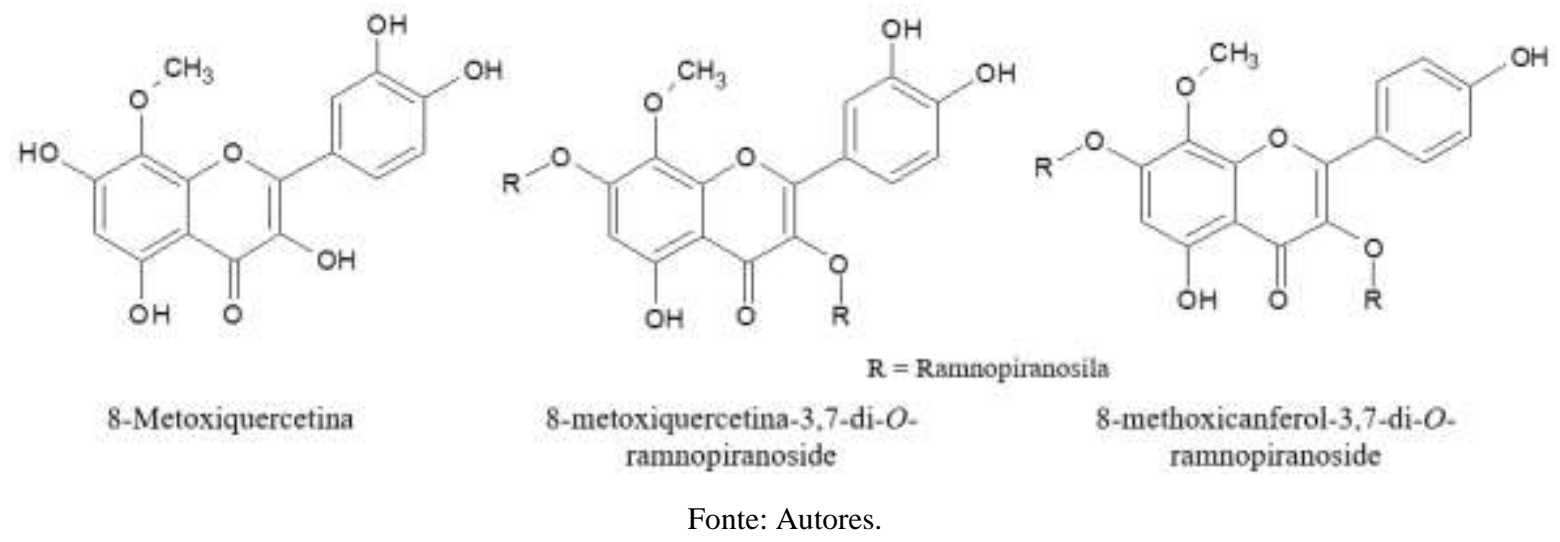

Martins et al. (2018b) pesquisaram sobre ação de inibição da AChE pelos extratos etanólicos de folhas, caule e látex de Pinhão-Roxo (Jatropha gossypifolia - Euphorbiaceae), no teste de CCD obteve resultado positivo para os três extratos, porém apenas o extrato etanólico das folhas $\left(\mathrm{CI}_{50}=24,51 \pm 0,21 \mu \mathrm{g} \cdot \mathrm{mL}^{-1}\right)$ mostrou-se relevante no ensaio de microdiluição quando comparado ao resultado do padrão de fisostigmina $\left(\mathrm{CI}_{50}=19,53 \pm 0,08 \mu \mathrm{g} \cdot \mathrm{mL}^{-1}\right)$. O principal constituinte isolado da planta com atividade anticolinesterásica foi o alcaloide glicosídeo do Jatropha (Figura 3).

Figura 3. Principal alcaloide caracterizados em J. gossypifolia.

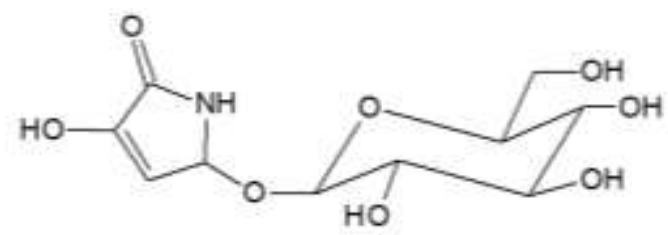

Glicosideo do Jatropha

Fonte: Autores. 
Penido et al. (2017) através do ensaio de diluição em microplaca, avaliou a inibição da AChE por 12 extratos etanólicos de plantas das famílias Anacardiaceae, Apocynaceae, Arecaceae, Fabaceae, Malvaceae e Myrtaceae. 6 extratos foram considerados como potentes inibidores de AChE: Mangabeira (Hancornia speciosa - Apocynaceae) com $\mathrm{CI}_{50}=10,13 \pm$ 0,17 $\mu \mathrm{g} \cdot \mathrm{mL}^{-1}$, Aroeira-do-Sertão (Myracrodruon urundeuva - Anacardiaceae) com $\mathrm{CI}_{50}=10,75 \pm 0,15 \mu \mathrm{g} \cdot \mathrm{mL}^{-1}$, Copaíba (Copaifera langsdorffii - Fabaceae) com $\mathrm{CI}_{50}=14,86 \pm 1,79 \mu \mathrm{g} \cdot \mathrm{mL}^{-1}$, Barbatimão (Stryphnodendron coriaceum - Fabaceae) com $\mathrm{CI}_{50}=17,56 \pm 0,50 \mu \mathrm{g} \cdot \mathrm{mL}^{-1}$, Goiabeira (Psidium guajava - Myrtaceae) com $\mathrm{CI}_{50}=18,98 \pm 0,11 \mu \mathrm{g} \cdot \mathrm{mL}^{-1}$ e Mangueira (Mangifera indica - Anacardiaceae) com $\mathrm{CI}_{50}=29,67 \pm 0,42 \mu \mathrm{g} \cdot \mathrm{mL}^{-1}$. O controle positivo foi a eserina com $\mathrm{CI}_{50}=19,53 \pm$ 0,08 $\mu \mathrm{g} \cdot \mathrm{mL}^{-1}$.Na pesquisa com 30 espécies de plantas medicinais do Parque Estadual do Cocó em Fortaleza, Morais et al. (2021) avaliou a correlação entre as atividades antioxidantes e anti-AChE dos extratos etanólico, todas as espécies em questão apresentaram inibição, porém apenas 6 foram considerada com alto poder inibidor da $\mathrm{AChE}\left(\mathrm{CI}_{50}<20,0 \mu \mathrm{g} \cdot \mathrm{mL}^{-1}\right)$, esse espécies foram Cajueiro (Anacardium occidentale - Anacardiaceae) com $\mathrm{CI}_{50}=15,09 \pm 0,04 \mu \mathrm{g} \cdot \mathrm{mL}^{-1}$, Paineira $($ Ceiba pentandra - Malvaceae) com $\mathrm{CI}_{50}=15,87 \pm 0,02 \mu \mathrm{g} \cdot \mathrm{mL}^{-1}$, Mangue-Branco (Laguncularia racemosa - Combretaceae) com CI 50 $=10,95 \pm 0,02 \mu \mathrm{g} \cdot \mathrm{mL}^{-1}$, Mangueira (Mangifera indica - Anacardiaceae) com $\mathrm{CI}_{50}=11,71 \pm 0,02 \mu \mathrm{g} \cdot \mathrm{mL}^{-1}$, Aroeira-do-Sertão (Myracrodrum urundeuva - Anacardiaceae) com $\mathrm{CI}_{50}=13,35 \pm 0,00 \mu \mathrm{g} \cdot \mathrm{mL}^{-1}$ e Castanholeira (Terminalia catappa Combretaceae) com $\mathrm{CI}_{50}=11,82 \pm 0,12 \mu \mathrm{g} \cdot \mathrm{mL}^{-1}$.

A mangiferina é um dos constituintes mais abundantes nas cascas e folhas da $M$. indica e mostrou uma ação de inibição dose dependente da $\mathrm{AChE}\left(\mathrm{CI}_{50}=62.8 \mu \mathrm{M}\right)$ (Jung et al, 2009). Outro estudo in vivo foi conduzido para avaliar o efeito neurofarmacológico da mangiferina na colinesterase cerebral e aminas biogênicas cerebrais juntamente com seu status antioxidante. O pré-tratamento com mangiferina restaurou os danos causados no cérebro inteiro dos animais devido ao uso da escopolamina como o aumento da acetilcolinesterase, a peroxidação lipídica e a redução da glutationa. Os autores afirmam que os efeitos observados da mangiferina sugerem utilizá-la no tratamento da Doença de Alzheimer (Biradar et al., 2012). A Figura 4 mostra a estrutura da mangiferina.

Figura 4. Representação estrutural da Mangiferina obtida da M. indica.

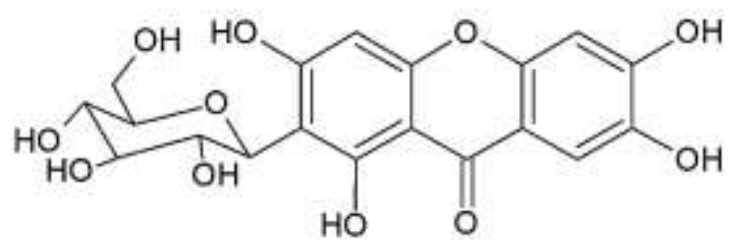

Fonte: Autores.

Lima et al. (2012), em seu estudo com o óleo essencial das folhas de Pitanga-Preta (Eugenia sulcata - Myrtaceae) mostraram que este foi capaz de inibir a ação da enzima acetilcolinesterase com um $\mathrm{CI}_{50}=4,66 \pm 0,48 \mu \mathrm{g} \cdot \mathrm{mL}^{-1}$. O controle positivo (fisostigmina) mostrou um $\mathrm{CI}_{50}=0,59 \pm 0,02 \mu \mathrm{g} \cdot \mathrm{mL}^{-1}$.

Dalai et al. (2014) em sua pesquisa com o extrato metanólico e o óleo essencial das folhas de canela (Cinnamomum zeylanicum - Lauraceae) obtiveram $\mathrm{CI}_{50}$ de 77,78 $\pm 0,03$ e 45,88 $\pm 1,94 \mu \mathrm{g} \cdot \mathrm{mL}^{-1}$ respectivamente, o padrão galantamina apresentou $\mathrm{CI}_{50}=22,34 \pm 0,56 \mu \mathrm{g} \cdot \mathrm{mL}^{-1}$. 
A pesquisa de Feitosa et al. (2017) com o óleo essencial das folhas de Cagaita (Eugenia dysenterica - Myrtaceae), mostrou uma redução da atividade da $\mathrm{AChE}$ com resultado significativo $\left(\mathrm{CI}_{50}=0,92 \mu \mathrm{g} \cdot \mathrm{mL}^{-1}\right)$ em comparação a rivastigmina $\left(\mathrm{CI}_{50}=1,87 \mu \mathrm{g} \cdot \mathrm{mL}^{-1}\right)$.

O trabalho de Martins et al. (2018a) com extrato hidroetanólico, aquoso, hexânico e clorofórmico de cascas de Catuaba (Trichilia catigua - Meliaceae), revelou atividade inibitória da AChE in vitro, sendo o extrato hidroalcoólico o mais potente com $\mathrm{CI}_{50}=142,0 \mu \mathrm{g} \cdot \mathrm{mL}^{-1}$. O controle positivo rivastigmina apresentou $\mathrm{CI}_{50}=18,0 \mu \mathrm{g} \cdot \mathrm{mL}^{-1}$.

No estudo de Nascimento et al. (2018), os autores avaliaram a capacidade de inibição da AChE com extratos etanólico, hexânico e aquoso de folhas, galhos e sementes de Batiputá (Ouratea fieldingiana - Ochnaceae), quando comparados o alcaloide padrão fisostigmina $\left(\mathrm{CI}_{50}=1,15 \pm 0,05 \mu \mathrm{g} \cdot \mathrm{mL}^{-1}\right)$ o extrato das folhas mostrou a melhor ação anticolinesterásica $\left(\mathrm{CI}_{50}=0,82 \pm 0,01 \mu \mathrm{g} \cdot \mathrm{mL}^{-1}\right)$, seguida do extrato aquoso das sementes $\left(\mathrm{CI}_{50}=9,19 \pm 0,03 \mu \mathrm{g} \cdot \mathrm{mL}^{-1}\right)$ e do extrato etanólico dos galhos $\left(\mathrm{CI}_{50}=11,89 \pm 0,05 \mu \mathrm{g} \cdot \mathrm{mL}^{-1}\right)$.

Pereira et al. (2018) em seu trabalho com óleo essencial das folhas de Jaramataia (Vitex gardneriana - Lamiaceae) mostrou que diferentes extrações do óleo possui um ótima inibição da $\mathrm{AChE}$ no teste de microdiluição com $\mathrm{CI}_{50}$ variando de 13,7 a $11,0 \mu \mathrm{g} \cdot \mathrm{mL}^{-1}$.

A pesquisa realizada por Dantas et al. (2020), com o extrato etanólico das sementes de Pitomba (Talisia esculenta Sapindaceae) mostrou que a inibição da $\mathrm{AChE}$ foi de $\mathrm{CI}_{50}=12,60 \pm 0,11 \mu \mathrm{g} \cdot \mathrm{mL}^{-1}$, quando comparado ao padrão fisostigmina $\left(\mathrm{CI}_{50}=1,15 \pm 0,55 \mu \mathrm{g} \cdot \mathrm{mL}^{-1}\right)$, esse resultado é considerado de alto potencial de inibição.

Barbosa et al. (2020), em sua pesquisa sobre a inibição da AChE pelo óleo essencial extraído das cascas de Cambiuva (Myrciaria floribunda - Myrtaceae) obteve o resultado de $\mathrm{CI}_{50}=0,08 \mu \mathrm{g} \cdot \mathrm{mL}^{-1}$, valor que torna a concentração inibitória mediana quando comparado ao padrão de neostigmina $\left(\mathrm{CI}_{50}=23,3 \mu \mathrm{g} \cdot \mathrm{mL}^{-1}\right)$ utilizado no ensaio. Em estudo de docking molecular os constituintes sesquiterpênicos $\delta$-cadineno e $\gamma$-cadineno do óleo essencial foram os mais ativos contra a proteínaalvo da AChE, utilizada como alvo para estudos computacionais de compostos contra Doença de Alzheimer. A Figura 5 mostra a estrutura dos dois compostos.

Figura 5. Compostos ativos em Docking presentes na M. florybunda.<smiles>CC1=CC2C(=C(C)CCC2C(C)C)CC1</smiles>

$\delta$-cadineno<smiles>C=C1CCC(C(C)C)C2C=C(C)CCC12</smiles>

$\gamma$-cadineno

Fonte: Autores.

Gasca et al. (2020), em sua pesquisa com extrato etanólico dos bulbos de Açucena (Hippeastrum psittacinum Amaryllidaceae), concluiu que ela possui uma atividade anticolinesterásica promissora $\left(\mathrm{CI}_{50}=11,2 \pm 0,7 \mu \mathrm{g} \cdot \mathrm{mL}^{-1}\right)$ quando comparado ao padrão galantamina $\left(\mathrm{CI}_{50}=0,16 \pm 0,01 \mu \mathrm{g} \cdot \mathrm{mL}^{-1}\right)$.

Estudo realizado em microplaca por Lima et al. (2021), como óleos essenciais extraídos das folhas de Capim-Limão (Cymbopogon flexuosus - Poaceae), flores de Gerânio (Pelargonium x ssp - Geraniaceae) e resina de Copaíba (Copaifera officinalis - Fabaceae) revelou resultados significativos quando comparados ao padrão de fisostigmina $\left(\mathrm{CI}_{50}=1,15 \mu \mathrm{g} \cdot \mathrm{mL}^{-1}\right)$, 
Research, Society and Development, v. 11, n. 1, e6211124262, 2022

(CC BY 4.0) | ISSN 2525-3409 | DOI: http://dx.doi.org/10.33448/rsd-v11i1.24262

sendo o $\mathrm{CI}_{50}$ do óleo de essencial de Cymbopogon flexuosus o melhor $\left(\mathrm{CI}_{50}=11,92 \mu \mathrm{g} \cdot \mathrm{mL}^{-1}\right)$, seguido do óleo de essencial de Copaifera officinalis $\left(\mathrm{CI}_{50}=12,0 \mu \mathrm{g} \cdot \mathrm{mL}^{-1}\right)$ e do óleo essencial de Pelargonium $x$ ssp $\left(\mathrm{CI}_{50}=13,05 \mu \mathrm{g} \cdot \mathrm{mL}^{-1}\right)$.

O estudo de Oliveira et al. (2021), revelou que os extratos etanólicos da Pata-de-Vaca (Bauhinia monandra Fabaceae) possui uma atividade anticolinesterásica com potencial moderado (folha: $\mathrm{CI}_{50}=25,38 \pm 0,37 \mu \mathrm{g} \cdot \mathrm{mL}^{-1}$, flores: $\mathrm{CI}_{50}=$ $23,23 \pm 0,48 \mu \mathrm{g} \cdot \mathrm{mL}^{-1}$ e galhos: $\mathrm{CI}_{50}=49,66 \pm 0,15 \mu \mathrm{g} \cdot \mathrm{mL}^{-1}$ ), o padrão fisostigmina apresentou $\mathrm{CI}_{50}=1,15 \pm 0,55 \mu \mathrm{g} \cdot \mathrm{mL}^{-1}$.

$\mathrm{Na}$ pesquisa realizada por Carvalho et al. (2021) com o óleo essencial extraído das vargens de Feijão-de-Boi (Cynophalla flexuosa - Capparaceae) os autores obtiveram o $\mathrm{CI}_{50}=7,9 \mu \mathrm{g} \cdot \mathrm{mL}^{-1}$ um resultado bom quando comparado ao padrão de fisostigmina $\left(\mathrm{CI}_{50}=1,2 \mu \mathrm{g} \cdot \mathrm{mL}^{-1}\right)$.

Frota et al. (2021) no estudo com o óleo extraído por fervura das sementes de Batiputá (Ouratea fieldingiana Ochnaceae) observou uma inibição da acetilcolinesterase de $\mathrm{CI}_{50}=12,58 \pm 0,10 \mu \mathrm{g} \cdot \mathrm{mL}^{-1}$, o padrão utilizado para comparação foram a galantamina com $\mathrm{CI}_{50}=1,02 \pm 0,02 \mu \mathrm{g} \cdot \mathrm{mL}^{-1}$.

Fernandes et al. (2021) na sua pesquisa com extratos hexânicos, metanólicos e etanólicos das folhas e sementes de faveira (Parkia platycephala) observaram que todas as amostras inibiram a $\mathrm{AChE}$, em comparação com o controle fisostigmina $\left(\mathrm{CI}_{50}=1,15 \pm 0,05 \mu \mathrm{g} \cdot \mathrm{mL}^{-1}\right)$, os valores de $\mathrm{CI}_{50}$ variaram de 9,85 a 15,68 $\mu \mathrm{g} \cdot \mathrm{mL}^{-1}$ destacando-se o extrato hexânico das sementes com 77,4\% de inibição da AChE.

Trabalho desenvolvido por Santos et al. (2012), revelou que o extrato aquoso das folhas de Mulungu (Erythrina velutina - Fabaceae) contém ação inibitória contra $\mathrm{AChE}$ no cérebros de camundongos nos teste in vitro com $\mathrm{CI}_{50}=0,57$ mg.mL $L^{-1}$ com inibição máxima $\left(\mathrm{E}_{\text {máx }}\right)$ de $89,0 \pm 1,5 \%$, no testes ex vivo para a dose de $400 \mathrm{mg} \cdot \mathrm{kg}^{-1} \mathrm{o}$ extrato apresentou inibição de 32,2 $\pm 3,9 \%$ contra a colinesterase cortical.

Oliveira et al. (2019) em sua pesquisa com o extrato etanólico das folhas de Lippia hirta (Verbenaceae) obtiveram uma inibição contra a AChE de $65 \%$ para a concentração de $0,1 \mathrm{mg} \cdot \mathrm{mL}^{-1}$ com $\mathrm{CI}_{50}=43,2 \mu \mathrm{g} \cdot \mathrm{mL}^{-1}$.

Guerra et al. (2021), no estudo da inibição da AChE com 4 diferentes óleos essenciais do Marmeleiro-Preto (Croton blanchetianus - Euphorbiaceae), revelou bons resultados em comparação com ao padrão fisostigmina $\left(\mathrm{CI}_{50}=1,15 \pm 0,05\right.$ $\left.\mu \mathrm{g} \cdot \mathrm{mL}^{-1}\right)$, porém com diferenças significativas entre eles: óleo essencial $1\left(\mathrm{CI}_{50}=10,31 \pm 0,03 \mu \mathrm{g} \cdot \mathrm{mL}^{-1}\right)$, óleo essencial $2\left(\mathrm{CI}_{50}\right.$ $\left.=15,53 \pm 0,03 \mu \mathrm{g} \cdot \mathrm{mL}^{-1}\right)$, óleo essencial $3\left(\mathrm{CI}_{50}=6,59 \pm 0,19 \mu \mathrm{g} \cdot \mathrm{mL}^{-1}\right)$, óleo essencial $4\left(\mathrm{CI}_{50}=11,98 \pm 0,53 \mu \mathrm{g} \cdot \mathrm{mL}^{-1}\right)$.

\section{Estudos da atividade anticolinesterásica com percentual de inibição e por cromatografia em camada delgada}

Na pesquisa desenvolvida por Frias et al. (2011) com os extratos hexânico, clorofórmico, metanólico e de acetato de etila de folhas de Liana (Banisteriopsis anisandra - Malpighiaceae) os autores obtiveram o resultado positivo para inibição da AChE no teste de Cromatografia de Camada Delgada (CCD) para o extrato metanólico.

Estudos realizados em microplaca por Mota et al. (2012) com extratos etanólicos e aquosos de 9 espécies das famílias Celastraceae, Cercideae, Chrysobalanaceae, Fabaceae, Moringaceae, Sapotaceae, Verbanaceae, e Viscaceae identificaram que no ensaio de inibição da $\mathrm{AChE}$ pelo método de microdiluição, o extrato etanólico das folhas de Alecrim (Hyptis fruticosa Lamiaceae) e os extratos aquoso e etanólico de entrecascas de Bom-Nome (Maytenus rígida - Celastraceae) apresentaram uma baixa atividade inibitória com percentual de inibição de $11 \%, 4 \%$ e $7 \%$ respectivamente. Já o extrato etanólico de sementes Moringa (Moringa oleifera - Moringaceae) e aquoso de folhas de Erva-de-Passarinho (Phoradendron piperoides - Viscaceae) apresentaram uma atividade inibitória considerada moderada com $40 \%$ e $47 \%$ de inibição respectivamente, porém o extrato aquoso de folhas de Agno-Casto (Vitex agnus-castus - Lamiaceae) obteve 74\% de inibição da AChE, caracterizando-se como uma potente atividade inibitória. 
Research, Society and Development, v. 11, n. 1, e6211124262, 2022

(CC BY 4.0) | ISSN 2525-3409 | DOI: http://dx.doi.org/10.33448/rsd-v11i1.24262

Onema et al. (2012), em sua pesquisa sobre a inibição da AChE em CCD utilizando extratos etanólicos de pele, polpa e sementes de dos frutos de Jenipapo (Genipa americana - Rubiaceae), Cirigüela (Spondias âmara - Anacardiaceae) e Umbu (Spondia tuberosa - Anacardiaceae) observaram quem o extrato de polpa de Genipa americana $(0,8 \pm 0,01 \mathrm{~mm})$ e das Spondias purpúrea $(0,9 \pm 0,01 \mathrm{~cm})$ apresentaram uma zona de inibição de AChE semelhante a do carbacol utilizado com controle positivo $(1,0 \pm 0,01 \mathrm{~cm})$, já as sementes de Spondia tuberosa apresentou zona de inibição razoável com $0,5 \pm 0,01 \mathrm{~cm}$.

Nos ensaio realizados com extratos etanólicos de folhas e galhos de 20 espécies de plantas da família Lauraceae, Yamaguchi et al. (2012) obtiveram resultados positivos para inibição da AChE no teste em CCD para 10 das espécies estudadas: extrato de galhos de Louro-Amarelo (Aniba panurensis), extrato de folhas de Eucalipto-Limão (Endlicheria citriodora), extrato de galhos de Louro-Pirarucu (Licaria cannella), extrato de galhos Louro-Chumbo (Licaria martiniana), extrato de galhos de Canela (Ocotea ceanothifolia), extrato de galhos de Louro (Ocotea leucoxylon), extrato de folhas e galhos de Louro-Preto (Ocotea minor), extrato de folhas de Louro-Fofo (Rhodostemonodaphne negrensis), extrato de folhas de Louro (Rhodostemonodaphne parvifolia), extrato de folhas de Louro (Rhodostemonodaphne recurva).

Estudo realizado com extrato etanólico das flores de Margarida (Bellis perennis - Asteraceae) Marques et al. (2013) indicou a inibição da $\operatorname{AChE}(81,83 \%)$ em camundongos após o tratamento com as doses de 50 mg.kg-1 $(1,91 \pm 0,25), 100$ mg.kg-1 $(1,66 \pm 0,21)$ e $150 \mathrm{mg} \cdot \mathrm{kg}^{-1}(1,79 \pm 0,13)$ em relação ao controle negativo $(10,03 \pm 0,16)$. De mesma forma quando comparado ao controle positivo, tratado com rivastigmina $(5,69 \pm 1,20)$ a redução foi $67,71 \%$.

Morais et al. (2013) em seu estudo sobre a correlação do teor de compostos fenólicos com a ação de inibição da AChE obtiveram resultados positivos para 10 dos 18 extratos etanólicos de folhas testados no ensaio com CCD, são eles: 0,7 cm para Torém (Cecropia pachystachya - Urticaceae), 0,6 cm para Canela-de-Cunhã (Croton zehntneri - Euphorbiaceae), 0,5 cm para Pitanga (Eugenia uniflora - Myrtaceae), 0,8 cm para Acerola (Malpighia glabra - Malpighiaceae), 0,7 cm para Erva-Cidreira (Lippia alba - Verbenaceae), 0,7 cm para Alecrim-de-Tabuleiro (Lippia microphylla - Verbenaceae), 0,7 cm para Alfavaca (Ocimum gratissimum - Lamiaceae), 0,6 cm para Quebra-Pedra (Phyllanthus amarus - Phyllanthaceae), 1,0 cm para FalsoBoldo (Plectranthus âmara - Phyllanthaceae) e 0,6 cm para Cajá (Spondias mombin - Anacardiaceae), destacando-se a L. alba e $P$. âmara como potenciais fontes de substâncias contra DA.

A pesquisa realizada por Farias et al. (2013) com 21 extratos etanólicos de sementes de diferentes espécies de plantas, revelou que 11 extratos apresentaram inibição da AChE no testes de diluição em microplaca de 96 poços: 54,1\% para AngicoVermelho (Anadenanthera macrocarpa - Fabaceae), 63,4\% para Pequí (Caryocar coriaceum - Caryocaraceae), 91,9\% para Araruta (Connarus detersus - Connaraceae), 13,9\% para Oiti (Licania tomentosa - Chrysobalanaceae), 52,4\% para Oiticica (Licania rígida - Chrysobalanaceae), 71,5\% para Faveira (Parkia platycephala - Fabaceae), 50,2\% para Catanduva (Piptadenia moniliformis - Fabaceae), 58,6\% para Sabão-de-Soldado (Sapindus saponária - Sapindaceae), 61,2\% para Pitomba (Talisia esculenta - Sapindaceae), 79,8\% para Pajeú (Triplaris gardneriana - Polygonaceae) e 49,4\% para Juazeiro (Ziziphus joazeiro - Rhamnaceae).

Silva et al. (2014a) estudaram o efeito dos óleos extraídos das partes aéreas (folhas e galhos finos) de 3 espécies de piper (Piper hispidum, Piper aleyreanum, e Piper anonifolium) pertencentes a família Piperaceae, os ensaios com CCD revelou que os óleos de $P$. Anonifolium e $P$. Hispidum ambos com $\mathrm{DL}=0,01 \mu \mathrm{g}$ contém uma ação anticolinesterásica bastante significativa, mostrando-se ser 100 vezes mais ativo do que a fisostigmina $(\mathrm{DL}=1,0 \mu \mathrm{g})$, como inibidores anticolinesterásicos.

No trabalho desenvolvido por Silva et al. (2014b) com o extrato etanólico das folhas do Limãozinho (Zanthoxylum syncarpum - Rutaceae) os autores obtiveram uma moderada atividade de inibição da AChE com 0,6 cm na placa de CCD em comparação ao padrão fisostigmina que foi de $0,9 \mathrm{~cm}$. 
Research, Society and Development, v. 11, n. 1, e6211124262, 2022

(CC BY 4.0) | ISSN 2525-3409 | DOI: http://dx.doi.org/10.33448/rsd-v11i1.24262

Santos et al. (2015) utilizando o óleo essencial das flores de Camará (Lantana âmara - Verbenaceae) conseguiram um alta inibição da acetilcolinesterase no ensaio de microdiluição, com um resultado de 77,15\%.

Segundo Silva et al. (2015) em seu trabalho com entrecascas e sementes de Araticum-do-Brejo (Annona Glabra Fabaceae), obtiveram resultados positivos no teste de CCD para todos os extratos produzidos (hexano, clorofórmio, acetado de etila e metanol), os valores variaram entre 0,6 a $0,8 \mathrm{~cm}$ sendo os halos medidos e comparados com o padrão positivo de fisostigmina que obteve $0,9 \mathrm{~cm}$.

Formagio et al. (2015) pesquisou sobre a ação de inibição da AChE por extratos metanólicos de folhas, sementes e capítulos florais de 5 espécies de Annonaceae: Araticum (Annona crassiflora), Fruta-do-Conde (Annona coriácea), Pinha (Annona sylvatica), Araticum-Cagão (Annona cacans) e Ata-Brava (Duguetia furfuracea), seu estudo revelou que no ensaio de diluição em microplaca apenas os extratos de folhas da A. cacans, sementes de A. sylvatica e capítulo floral de A. coriácea e D. furfuracea apresentaram resultados negativos, no ensaio de CCD apenas a espécie $A$. cacans não apresentou halo de inibição contra AChE.

No trabalho desenvolvido por Calixto Júnior et al. (2015) com os extratos etanólicos das folhas e cascas de Cuiteleiro (Prockia crucis - Salicaceae) os autores observaram um halo de inibição da AChE de 0,8 e 1,0 cm respectivamente no ensaio desenvolvido por CCD, resultado semelhante ao do padrão fisostigmina $(0,9 \mathrm{~cm})$.

Na pesquisa realizada com extratos de folhas e galhos de Caroba (Jacaranda oxyphylla - Bignoniaceae) Pereira et al. (2015) obtiveram resultados promissores na inibição da AChE no ensaio de microdiluição, o extrato hexano das folhas e o extrato hexânico e etanólico dos galhos apresentaram atividades de 71,5 à 84,1\% em comparação com os efeitos inibitórios do padrão fisostigmina $(97,2 \%)$.

Paredes et al. (2016), na avaliação da potência de inibição da AChE pelos extratos metanólicos de folhas, raiz e casca da raiz de Faveleira (Cnidoscolus quercifolius - Euphorbiaceae), obtiveram um resultado significativo no teste de CCD, os extratos de folhas e casca de raiz obtiveram um halo de inibição de $0,8 \mathrm{~cm}$ e o extrato de raiz um halo de $0,7 \mathrm{~cm}$, o padrão fisostigmina teve um halo de inibição de $0,9 \mathrm{~cm}$.

O estudo de Morais et al. (2017) com o extrato etanólico de folhas de Mutamba (Guazuma ulmifolia - Malvaceae) no teste de CCD revelou uma atividade de inibição contra AChE muito significativa, com uma zona de inibição de crescimento de $1,0 \mathrm{~cm}$, próximo do resultado obtido pelo padrão de fisostigmina que foi de $0,9 \mathrm{~cm}$.

Alves et al. (2017) em sua pesquisa com extratos etanólicos de casca e polpa do Pequí (Caryocar coriaceum Caryocaraceae) obtiveram um halo de inibição de 0,85 e $0,9 \mathrm{~cm}$ na placa de CCD, resultado com uma alta inibição da acetilcolinesterase, o padrão positivo foi a fisostigmina com halo de inibição de $0,9 \mathrm{~cm}$. A isoquercitrina foi o principal constituinte caracterizados nos extratos (Figura 6). 
Figura 6. Composto caracterizado no extrato da Caryocar coriaceum.<smiles>O=c1c(OC2OC(CO)C(O)C(O)C(O)C2O)c(-c2ccc(O)c(O)c2)oc2cc(O)cc(O)c12</smiles>

Isoquercitrina

Fonte: Autores.

Na pesquisa de Santos et al. (2018) com extratos de folhas de Capim-Amargoso (digitaria insularis - Poaceae) os autores obtiveram resultados moderados na inibição da AChE com os extratos hexânico e de acetato de etila (30-50\% de inibição) quando comparado ao padrão fisostigmina (99\%).

O estudo de Ribeiro et al. (2019) com óleo extraído das sementes de Caneleira-Verdadeira (Cinnamomum zeylanicum Lauraceae) mostrou-se com uma alta capacidade de inibição da AChE com 65,5\% no teste de microdiluição.

Na pesquisa de Pedroso et al. (2019) com extrato metanólico da raiz de Algodão-do-Campo (Cochlospermum regium Bixaceae) apresentou resultados significativos no teste in vivo em ratos Wistar, o grupo tratado com $100 \mathrm{mg}^{\mathrm{k}} \mathrm{kg}^{-1}$ apresentou inibição para AChE no córtex cerebral em 69\%, no hipocampo em 53,76\% e de 42,20\% no hipotálamo.

Após análises das pesquisas citadas neste estudo, nota-se que este levantamento mostrou que 152 espécies de plantas da fauna brasileira tiveram estudos sobre seu potencial uso para o tratamento da Doença de Alzheimer. A Tabela 2 mostra a quantidade de espécies distribuídas por famílias botânicas mencionadas nos estudos.

Tabela 2. Quantidade de plantas brasileiras distribuídas por família botânica.

\begin{tabular}{|c|c|c|c|c|c|}
\hline Famílias Botânicas & Quantidade & Porcentagem & Famílias Botânicas & Quantidade & Porcentagem \\
\hline Amaranthaceae & 1 & 0,66 & Malvaceae & 3 & 1,97 \\
\hline Amaryllidaceae & 1 & 0,66 & Meliaceae & 1 & 0,66 \\
\hline Anacardiaceae & 8 & 5,26 & Moraceae & 2 & 1,32 \\
\hline Annonaceae & 6 & 3,95 & Moringaceae & 1 & 0,66 \\
\hline Apocynaceae & 1 & 0,66 & Myrtaceae & 7 & 4,61 \\
\hline Arecaceae & 1 & 0,66 & Nyctaginaceae & 1 & 0,66 \\
\hline Asteraceae & 1 & 0,66 & Ochnaceae & 1 & 0,66 \\
\hline Bignoniaceae & 2 & 1,32 & Phyllanthaceae & 2 & 1,32 \\
\hline Bixaceae & 1 & 0,66 & Piperaceae & 3 & 1,97 \\
\hline Boraginaceae & 1 & 0,66 & Poaceae & 2 & 1,32 \\
\hline Capparaceae & 2 & 1,32 & Polygalaceae & 1 & 0,66 \\
\hline Caryocaraceae & 1 & 0,66 & Polygonaceae & 1 & 0,66 \\
\hline Celastraceae & 1 & 0,66 & Punicaceae & 1 & 0,66 \\
\hline Chrysobalanaceae & 3 & 1,97 & Rhamnaceae & 1 & 0,66 \\
\hline Combretaceae & 2 & 1,32 & Rubiaceae & 2 & 1,32 \\
\hline Connaraceae & 1 & 0,66 & Rutaceae & 2 & 1,32 \\
\hline Convolvulaceae & 2 & 1,32 & Salicaceae & 1 & 0,66 \\
\hline Crassulaceae & 3 & 1,97 & Sapindaceae & 2 & 1,32 \\
\hline Euphorbiaceae & 7 & 4,61 & Sapotaceae & 1 & 0,66 \\
\hline Fabaceae & 32 & 21,05 & Schrophulariaceae & 1 & 0,66 \\
\hline Geraniaceae & 1 & 0,66 & Sterculiceae & 1 & 0,66 \\
\hline Lamiaceae & 5 & 3,29 & Urticaceae & 1 & 0,66 \\
\hline Lauraceae & 21 & 13,82 & Verbenaceae & 6 & 3,95 \\
\hline Lecythidaceae & 1 & 0,66 & Viscaceae & 1 & 0,66 \\
\hline Malpighiaceae & 2 & 1,32 & & & \\
\hline
\end{tabular}

Fonte: Autores. 
Research, Society and Development, v. 11, n. 1, e6211124262, 2022

(CC BY 4.0) | ISSN 2525-3409 | DOI: http://dx.doi.org/10.33448/rsd-v11i1.24262

Todas as espécies de plantas estão distribuídas em 49 famílias botânicas, onde a família Fabaceae foi a mais citada com 32 espécies, correspondendo a 21,05\% do total, logo em seguida vem a família Lauraceae com 21 espécies representando 13,82\% e a família Anacardiaceae com 8 espécies e 5,26\% do total. As demais espécies não alcançaram 5\% de citação.

Em relação as partes das plantas utilizadas nos estudos notaram-se que as folhas foram as mais utilizada nas pesquisas (94 vezes), representando um total de 43,12\%, seguida das sementes (37 vezes) com 16,97\% e cascas do caule ( 22 vezes) com $10,09 \%$ do total pesquisado. Todas as partes utilizadas estão representadas na Figura 7.

Figura 7. Número de partes das plantas utilizadas nos estudos.

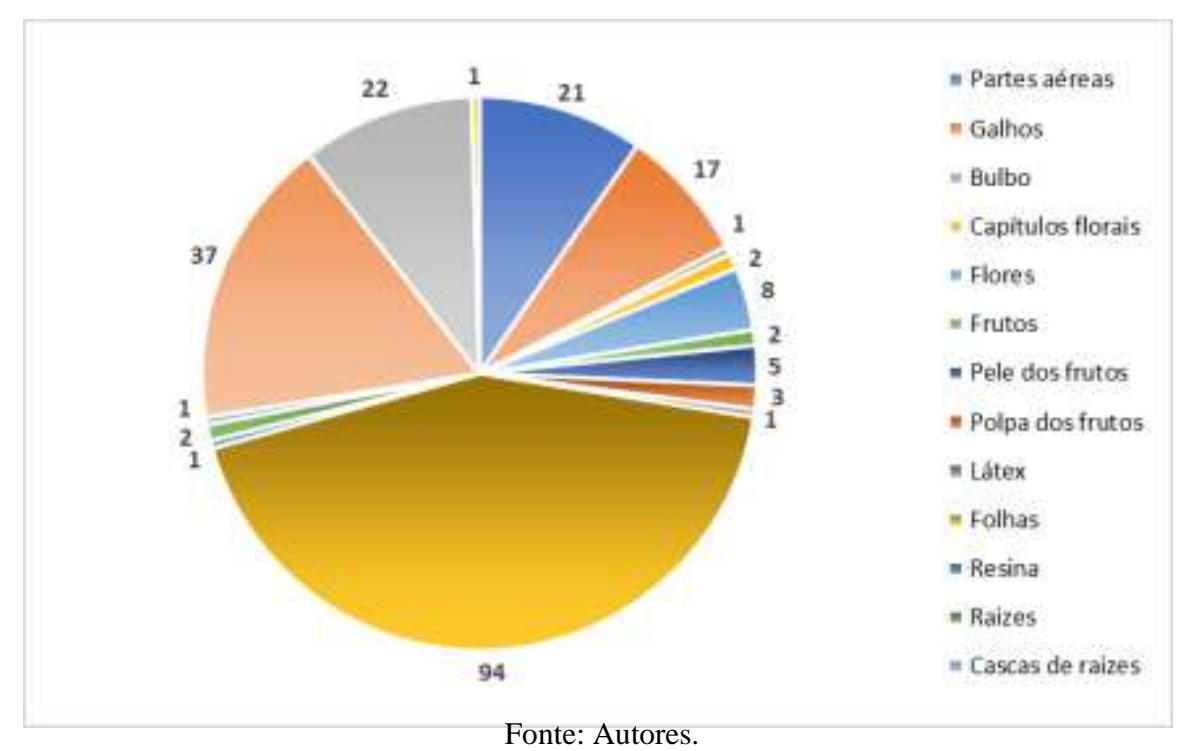

A grande maioria dos estudos utilizaram extratos para a realização das pesquisas, totalizando 253 amostras, apenas 17 utilizaram os óleos essenciais e 1 estudo utilizou o óleo fixo. As extrações por etanol se destacaram com 140 vezes (51,66\%), sendo seguida pelas realizadas com metanol (43 extrações ou 15,87\%) e pelas de acetato de etila com 28 extrações (10,33\%). A Figura 8 mostra a distribuição quantitativa de todas as extrações. 
Research, Society and Development, v. 11, n. 1, e6211124262, 2022

(CC BY 4.0) | ISSN 2525-3409 | DOI: http://dx.doi.org/10.33448/rsd-v11i1.24262

Figura 8. Tipo e número de extrações por processo.

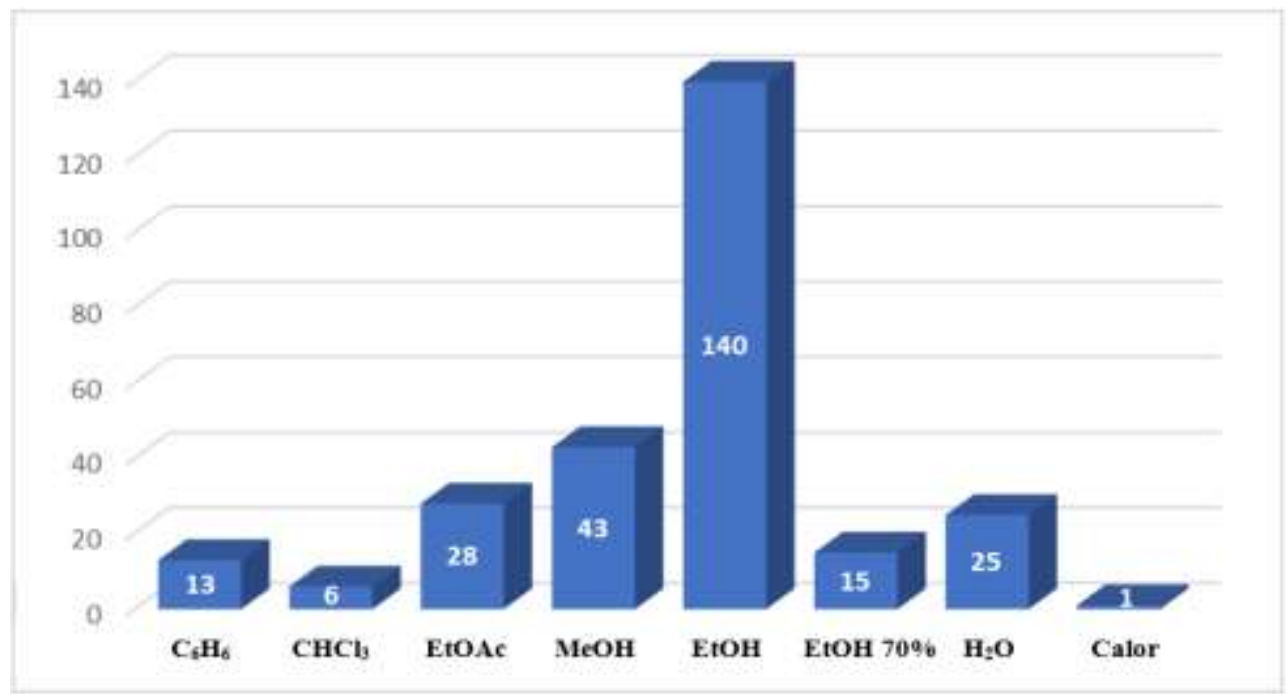

Legenda: $\mathrm{C}_{6} \mathrm{H}_{6}$ (Hexano), $\mathrm{CHCl}_{3}$ (Clorofórmio), EtOAc (Acetato de Etila), $\mathrm{MeOH}$ (Metanol), EtOH (Etanol), $\mathrm{H}_{2} \mathrm{O}$ (Água). Fonte: Autores.

A maior parte dos ensaios para avaliar a inibição da acetilcolinesterase foram determinados pelo o método espectrofotométrico de Ellman adaptado para diluição em microplaca de 96 poços, com 182 ensaios (54,17\%). Já o número de ensaios que utilizam o método de CCD foram de 151 (44,94\%) e os teste in vivo foram realizados apenas em 3 ensaios $(0,89 \%)$.

$\mathrm{Na}$ análise da intensidade promovida pelas espécies, observou-se que a maioria delas (73 espécies) demostraram uma alta intensidade de inibição, outras 30 obtiveram uma intensidade moderada e 7 resultaram em uma intensidade baixa, também observou-se que 31 espécies tiveram resultados negativos contra a $\mathrm{AChE}$ e que não foram possíveis mensurar a inibição de outras 11 espécies. A Figura 9 revela a porcentagem de espécies por intensidade de inibição da AChE para aquelas com resultados positivos.

Figura 9. Resultados da intensidade de inibição da $\mathrm{AChE} \mathrm{em} \mathrm{porcentagem.}$

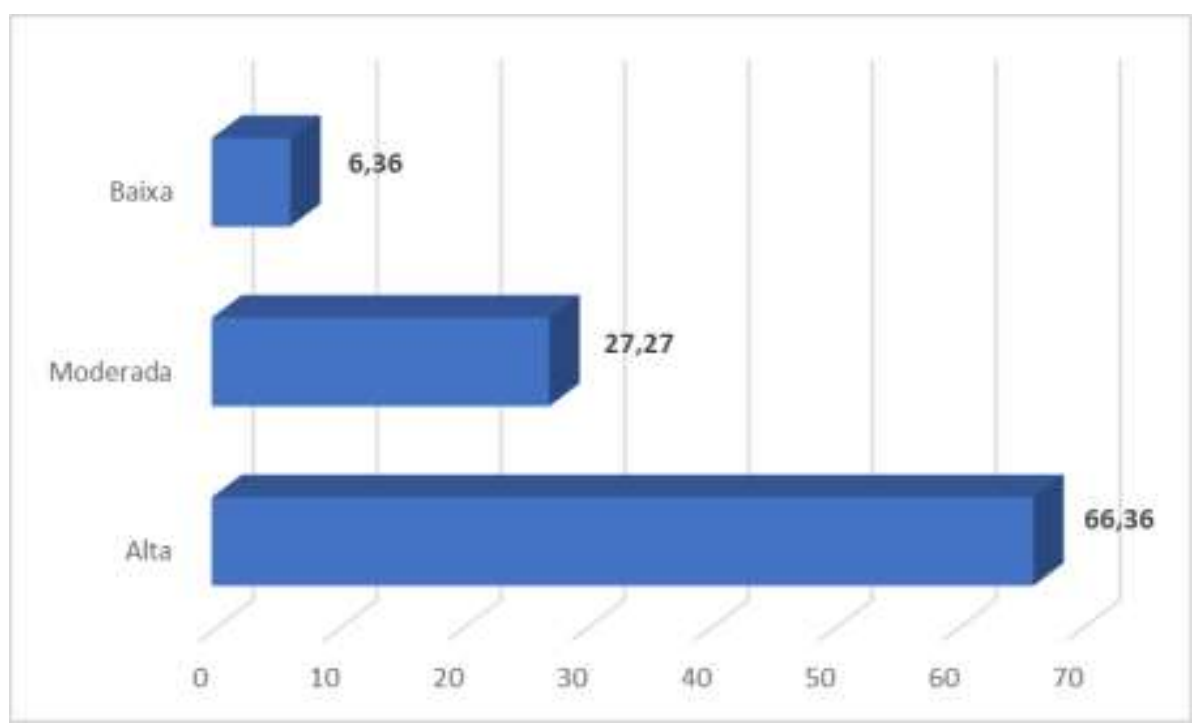

Fonte: Autores. 
Research, Society and Development, v. 11, n. 1, e6211124262, 2022

(CC BY 4.0) | ISSN 2525-3409 | DOI: http://dx.doi.org/10.33448/rsd-v11i1.24262

\section{Considerações Finais}

Após todas as análises, notou-se a crescente busca por substâncias inibidoras da enzima acetilcolinesterase (AChE) objetivando seus usos em tratamentos de doenças neurodegenerativas do sistema nervoso humano, em especial a Doença de Alzheimer, tal busca acarretou no aumento dos números das publicações envolvendo as pesquisas com as plantas da flora brasileira, contribuindo para formação de um banco de dados bastante significativo para as espécies vegetais do país.

De modo geral, o estudo demonstrou o potencial das plantas brasileiras contra a enzima AChE, sendo os extratos etanólicos das folhas o principal meio de estudos utilizados. Muitas das espécies foram consideradas ativas contra a enzima AChE, das 152 espécies relatadas 110 promoveram a inibição, sendo 48,03\% do total com uma alta intensidade, vale ressaltar que diante do tamanho da nossa flora, poucas espécies foram investigadas, tampouco foram testadas in vivo para se justificar os resultados obtidos nos ensaios in vitro.

Os dados obtidos nessa revisão sistemática abrem oportunidades para futuras pesquisas com as plantas da flora brasileira. Sugere-se que novas pesquisas sejam realizadas focando nos testes in vivo e contemplado as espécies de cada região ou bioma existente no Brasil.

\section{Referências}

Alves, D. R., Morais, S. M., Tomiotto-Pellissier, F., Miranda-Sapla, M. M., Vasconcelos, F. R., Silva, I. N. G., Sousa, H. A., Assolini, J. P., Conchon-Costa, I., \& Pavanelli, W. R. (2017). Flavonoid composition and biological activities of ethanol extracts of Caryocar coriaceum Wittm., a native plant from Caatinga Biome. Evidence-Based Complementary And Alternative Medicine, 2017, Article 6834218. http://dx.doi.org/10.1155/2017/6834218

Araújo, C. R. M., Santos, V. L. A., \& Gonsalves, A. A. (2016). Acetylcholinesterase - AChE: a pharmacological interesting enzyme. Revista Virtual de Química, 8(6), 1818-1834. http://dx.doi.org/10.21577/1984-6835.20160122

Barbosa, D. C. S., Holanda, V. N., Assis, C. R. D., Aguiar, J. C. R. O. F., Nascimento, P. H., Silva, W. V., Navarro, D. M. A. F., Silva, M. V., Lima, V. L. M., \& Correia, M. T. S. (2020). Chemical composition and acetylcholinesterase inhibitory potential, in silico, of Myrciaria floribunda (H. West ex Willd.) O. Berg fruit peel essential oil. Industrial Crops \& Products, 151(2020), 112372. https://doi.org/10.1016/j.indcrop.2020.112372

Biradar, S. M., Joshi, H., \& Chheda, T. K. (2012). Neuropharmacological effect of Mangiferin on brain cholinesterase and brain biogenic amines in the management of Alzheimer's disease. European journal of pharmacology, 683(1-3), 140-147. https://doi.org/10.1016/j.ejphar.2012.02.042

Calixto Júnior, J. T., Morais, S. M., Vieira, L. G., Alexandre, J. B., Costa, M. S., Morais-Braga, M. F. B., Júnior, J. E. G. L., Silva, M. M. O., Barros, L. M., \& Coutinho, H. D. M. (2015). Phenolic composition and anticholinesterase, antioxidant, antifungal and antibiotic modulatory activities of Prockia crucis (Salicaceae) extracts collected in the Caatinga biome of Ceará State, Brazil. European Journal of Integrative Medicine, 7(5), 547-555. https://doi.org/10.1016/j.eujim.2015.04.006

Carvalho, N. K. G., Camilo, C. J., Nonato, C. F. A., Leite, D. O. D., Rodrigues, F. F. G., Alves, D. R., Morais, S. M., \& Costa, J. G. M. (2021). Essential oil of Cynophalla flexuosa and its cytotoxicity, antioxidant, and anti-acetylcholinesterase effect. Chemistry of Natural Compounds, 57(3), 566-568. https://doi.org/10.1007/s10600-021-03418-7

Dalai, M. K., Bhadra, S., Chaudhary, S. K., Chanda, J., Bandyopadhyay, A., \& Mukherjee, P. K. (2014). Anticholinesterase activity of Cinnamomum zeylanicum L. leaf extract. CELLMED, 4(2), 11.1-11.6. https://doi.org/10.5667/tang.2013.0034

Dantas, L. V. B., Lopes, F. F. S., Alves, D. R., Frota, L. S., Cardoso, A. L. H., \& Morais, S. M. (2020). Avaliação fitoquímica, quantificação de fenóis e flavonóides totais, atividade antioxidante e antiacetilcolinesterase do extrato etanólico da Talísia esculenta (Pitomba). Brazilian Journal of Development, 6(8), 60597-60602. https://doi.org/10.34117/bjdv6n8-467

Deture, M. A., \& Dickson, D. W. (2019). The neuropathological diagnosis of Alzheimer's disease. Molecular Neurodegeneration, 14(1), 1-18. https://doi.org/10.1186/s13024-019-0333-5

Ellman, G. L., Courtney, K. D., Andres, V. J., \& Featherstone, R. M. (1961). A new and rapid colorimetric of acetylcholinesterase determination of acetylcholinesterase activity. Biochemical Pharmacology, 7(2), 88-95. https://dx.doi.org/10.1016/0006-2952(61)90145-9

Farias, D. F., Souza, T. M., Viana, M. P., Soares, B. M., Cunha, A. P., Vasconcelos, I. M., Ricardo, N. M. P. S., Ferreira, P. M. P., Melo, V. M. M., \& Carvalho, A. F. U. (2013). Antibacterial, antioxidant, and anticholinesterase activities of plant seed extracts from Brazilian semiarid region. Biomed Research International, 2013(1), Article 510736. http://dx.doi.org/10.1155/2013/510736

Feitosa, C. M., Freitas, R. M., Luz, N. N. N., Bezerra, M. Z. B., \& Trevisan, M. T. S. (2011). Acetylcholinesterase inhibition by some promising Brazilian medicinal plants. Brazilian Journal of Biology, 71(3), 783-789. https://doi.org/10.1590/S1519-69842011000400025 
Research, Society and Development, v. 11, n. 1, e6211124262, 2022

(CC BY 4.0) | ISSN 2525-3409 | DOI: http://dx.doi.org/10.33448/rsd-v11i1.24262

Feitosa, C. M., Barbosa, A. R., Melo, C. H. S., Freitas, R. M., Fontes, F. E. N., Costa, E. V., Rashed, K. N. Z., \& Costa Júnior, J. S. (2017). Antioxidant and anticholinesterase activities of the essential oil of Eugenia dysenterica DC. African Journal of Pharmacy and Pharmacology, 1(19), 241-249. https://doi.org/10.5897/AJPP2015.4438

Fernandes, R. M. N., Rodrigues, M. A. M., Panontin, J. F., Alves, D. R., Morais, S. M., Soares, I. M., \& Scapin, E. (2021). Chemical investigation, toxic potential and acetylcholinesterase inhibitory effect of Parkia platycephala leaf and seed extracts. Journal of Medicinal Plants Research, 15(9), 401-412. https://doi.org/10.5897/JMPR2021.7158

Formagio, A. S., Vieira, M. C., Volobuff, C. R., Silva, M. S., Matos, A. I., Cardoso, C. A., Foglio, M. A., \& Carvalho, J. E. (2015). In vitro biological screening of the anticholinesterase and antiproliferative activities of medicinal plants belonging to Annonaceae. Brazilian Journal of Medical and Biological Research, 48(4), 308-315, http://dx.doi.org/10.1590/1414-431X20144127.

Frias, U. A., Costa, M. C. M., \& Takahashi, J. A. (2011). Caracterização fitoquímica e avaliação das atividades antibacteriana e anticolinesterásica de extratos de Banisteriopsis anisandra A. Juss. (Malpighiaceae). Revista Cubana de Plantas Medicinales, 16(1), 60-71. http://scielo.sld.cu

Frota, L. S., Lopes, F. F. S., Alves, D. R., Freitas, L. S., Franco, G. M. G., \& Morais, S. M. de. (2021). Composição química e avaliação das atividades antioxidante e anticolinesterásica do óleo dos frutos de Ouratea fieldingiana (Gargner) Engl. Research, Society and Development, 10(10), e532101019013. https://doi.org/10.33448/rsd-v10i10.19013

Galvão, T. F., Pansani, T. S. A., \& Harrad, D. (2015). Principais itens para relatar revisões sistemáticas e meta-análises: A recomendação PRISMA. Epidemiologia e Serviços de Saúde, 24(2), 335-342. https://doi.org/10.5123/S1679-49742015000200017

Gasca, C. A., Moreira, N. C. S., Almeida, F. C., Gomes, J. V. D., Castillo, W. O., Fagg, C. W., Magalhães, P. O., Fonseca-Bazzo, Y. M., Sakamoto-Hojo, E., \& Medeiros, Y. K. (2020). Acetylcholinesterase inhibitory activity, anti-inflammatory, and neuroprotective potential of Hippeastrum psittacinum (Ker Gawl.) herb (Amaryllidaceae). Food And Chemical Toxicology, 145, Article 111703. https://doi.org/10.1016/j.fct.2020.111703

Guerra, D. L., Rodrigues, A. L. M., Alves, D. R., Silveira, E. R., Morais, S. M. (2021). Intraspecific variation of the chemical composition and antioxidant and anticholinesterase activities of essential oils and phenolic content of four Croton blanchetianus Baill specimens. In Morais, S. M. (Org.), Biotechnological potential of essential oils from native and cultivated plants in Brazil, (42-51). Editora Poisson. http://dx.doi.org/10.36229/978-65-5866-092-7.cap.03

Jung, K., Lee, B. Han, S. J., Ryu, J. H., Kim, D. H. (2009). Mangiferin Ameliorates Scopolamine-Induced Learning Deficits in Mice. Biological \& Pharmaceutical Bulletin, 32(2), 242-246. http://dx.doi.org/10.1248/bpb.32.242

Lima, B. G., Tietbohl, L. A. C., Fernandes, C. P., Cruz, R. A. S., Botas, G. S., Santos, M. G., Silva-Filho, M. V., \& Rocha, L. (2012). Chemical composition of essential oils and anticholinesterasic activity of Eugenia sulcata Spring ex Mart. Latin American Journal of Pharmacy, 1(31), 152-155. https://www.researchgate.net/publication/274836495

Lima, L. R., Andrade, F. K., Alves, D. R., Morais, S. M., \& Vieira, R. S. (2021). Anti-acetylcholinesterase and toxicity against Artemia salina of chitosan microparticles loaded with essential oils of Cymbopogon flexuosus, Pelargonium x ssp and Copaifera officinalis. International Journal of Biological Macromolecules, 167(1) 1361-1370. https://doi.org/10.1016/j.ijbiomac.2020.11.090

Magalhães, P. K. A., Araujo, E. N., Santos, A. M., Vanderlei, M. B., Souza, C. C. L., Correia, M. S., Fonseca, S. A., Pavão, J. M. J. S., Souza, M. A., Costa, J. G., Santo, A. F., \& Matos-Rocha, T. J. (2022). Ethnobotanical and ethnopharmacological study of medicinal plants used by a traditional community in Brazil's northeastern. Brazilian Journal of Biology, 82, e237642. https://doi.org/10.1590/1519-6984.237642

Marques, T. H. C., Santos, P. S., Freitas, R. M., Carvalho, R. B. F., Melo, C. H. S., David, J. P., David, J. M., \& Lima, L. S. (2013). Atividade anticolinesterásica e perfil químico de uma fração cromatográfica ativa do extrato etanólico das flores Bellis perennis L. (Asteraceae). Química nova, 36(4), 549-553. https://doi.org/10.1590/S0100-40422013000400012

Martins, G. V., Alves, D. R., Viera-Araújo, F. M., Rondon, F., Braz-Filho, R., \& Morais, S. M. (2018). Chemical study and evaluation of antioxidant, antiacetylcholinesterase and antileishmanial activities of extracts from Jatropha gossypifolia L. (Pião Roxo). Revista Virtual de Química, 10(1)21-36. http://dx.doi.org/10.21577/1984-6835.20180004

Martins, N. O., Brito, I. M., Araújo, S. S. O., Negri, G., Carlini, E. A., \& Mendes, F. R. (2018) Antioxidant, anticholinesterase and antifatigue effects of Trichilia catigua (Catuaba). BMC Complementary and Alternative Medicine, 18(1), 1-13. https://doi.org/10.1186/s12906-018-2222-9

Morais, S. M., Lima, K. S. B., Siqueira, S. M. C., Cavalcanti, E. S. B., Souza, M. S. T., Menezes, J. E. S. A., \& Trevisan, M. T. S. (2013). Correlation between antiradical, anti-acetylcholinesterase activities and total phenol content of medicinal plant extracts from live pharmacies. Revista Brasileira de Plantas Medicinais, 15(4), 575-582. https://dx.doi.org/10.1590/S1516-05722013000400014

Morais, S. M., Calixto-Júnior, J. T., Ribeiro, L. M., Sousa, H. A., Silva, A. A. S., Figueiredo, F. G., Matias, E. F. F., Boligon, A. A., Athayde, M. .L., MoraisBraga, M. F. B., \& Coutinho, H. D. M. (2017). Phenolic composition and antioxidant, anticholinesterase and antibiotic-modulating antifungal activities of Guazuma ulmifolia Lam. (Malvaceae) ethanol extract. South African Journal of Botany, 110 (2017), 251-257. http://dx.doi.org/10.1016/j.sajb.2016.08.003

Morais, S. M., Alves, D. R., Frota, L. S., Pinheiro, S. de O., Silva, A. C. S., \& Silva, W. M. B. (2020). Atividades antioxidantes e anticolinesterásicas do extrato das folhas de Jaramataia (Vitex gardneriana Schauer). Brazilian Journal of Development, 6(5), 28802-28810. https://doi.org/10.34117/bjdv6n5-358

Morais, S. M., Lopes, F. F. S., Fontenele, G. A., Silva, M. V. F., Fernandes, V. B., \& Alves, D. R. (2021). Total phenolic content and antioxidant and anticholinesterase activities of medicinal plants from the State's Cocó Park (Fortaleza-CE, Brazil). Research, Society And Development, $10(5)$, e7510514493. http://dx.doi.org/10.33448/rsd-v10i5.14493

Mota, W. M., Barros, M. L., Cunha, P. E. L., Santana, M. V. A., Stevam, C. S., Leopoldo, P. T. G., \& Fernandes, R. P. M. (2012). Avaliação da inibição da acetilcolinesterase por extratos de plantas medicinais. Revista Brasileira de Plantas Medicinais, 14(4), 624-628. https://dx.doi.org/10.1590/S151605722012000400008 
Research, Society and Development, v. 11, n. 1, e6211124262, 2022

(CC BY 4.0) | ISSN 2525-3409 | DOI: http://dx.doi.org/10.33448/rsd-v11i1.24262

Nascimento, J. E. T.; Rodrigues, A. L. M.; Lisboa, D. S.; Liberato, H. R.; Falcão, M. J. C.; Silva, C. R.; Nobre Júnior, H. V.; Braz Filho, R.; Paula Junior, V. F., \& Alves, D. R. (2018). Chemical composition and antifungal in vitro and in silico, antioxidant, and anticholinesterase activities of extracts and constituents of Ouratea fieldingiana (DC.) Baill. Evidence-Based Complementary And Alternative Medicine, 2018, Article 1748487, 1-12. https://doi.org/10.1155/2018/1748487

Oliveira, A. S., Zapp, E., Brondani, D., Hoppe, T. D., Meier, L., \& Brighente, I. M. C. (2019). Investigation of antioxidant activity, acute toxicity and anticholinesterasic potential of Lippia hirta (Verbenaceae). Revista Virtual de Química, 11(2), 432-448. https://rvqsub.sbq.org.br/index.php/rvq/article/view/3148

Oliveira, D. P., Cavalcanti, E. S. B., Morais, S. M., Pinto, C. C. C., Lopes, F. F. S., Rodrigues, A. L. M., Alves, D. R., \& Maia, A. I. V.. (2021). Perfil cromatográfico por HPLC-DAD, potencial antiacetilcolinesterase e toxicidade de extratos etanólicos da espécie Bauhinia Monandra. Brazilian Journal of Development, 7(1), 1183-1197. https://dx.doi.org/10.34117/bjdv7n1-080

Omena, C. M. B., Valentim, I. B., Guedes, G. S., Rabelo, L. A., Mano, C. M., Bechara, E. J. H., Sawaya, A. C. H. F., Trevisan, M. T. S., Costa, J. G., \& Ferreira, R. C. S. (2012). Antioxidant, anti-acetylcholinesterase and cytotoxic activities of ethanol extracts of peel, pulp and seeds of exotic brazilian fruits. Food Research International, 49(2012), 334-344. https://dx.doi.org/10.1016/j.foodres.2012.07.010

Paredes, P. F. M., Vasconcelos, F. R., Paim, R. T. T., Marques, M. M. M., Morais, S. M., Lira, S. M., Braquehais, I. D., Vieira, I. G. P., Mendes, F. N. P., \& Guedes, M. I. F. (2016). Screening of bioactivities and toxicity of Cnidoscolus quercifolius Pohl. Evidence-Based Complementary and Alternative Medicine, Article 7930563, 1-9. http://dx.doi.org/10.1155/2016/7930563

Patel, S. S., Raghuwanshi, R., Masood, M., Acharya, A., \& Jain, S. K. (2018). Medicinal plants with acetylcholinesterase inhibitory activity. Reviews in the Neurosciences, 29(5), 491-529. https://dx.doi.org/10.1515/revneuro-2017-0054

Pedroso, T. F. M., Bonamigo, T. R., Silva, J., Vasconcelos, P., Félix, J. M., Cardoso, C. A. L., Souza, R. I. C., Santos, A. C., Volobuff, C. R. F., \& Formagio, A. S. N. (2019). Chemical constituents of Cochlospermum regium (Schrank) Pilg. root and its antioxidant, antidiabetic, antiglycation, and anticholinesterase effects in wistar rats. Biomedicine \& Pharmacotherapy, 111, 1383-1392. https://doi.org/10.1016/j.biopha.2019.01.005

Penido, A. B., Morais, S. M., Ribeiro, A. B., \& Silva, A. Z. (2016). Ethnobotanical study of medicinal plants in Imperatriz, state of Maranhão, Northeastern Brazil. Acta Amazonica, 46(4), 345-354. https://doi.org/10.1590/1809-4392201600584

Penido, A. B., Morais, S. M., Ribeiro, A. B., Alves, D. R., Rodrigues, A. L. M., Santos, L. H., \& Menezes, J. E. S. A. (2017). Medicinal plants from northeastern Brazil against Alzheimer's disease. Evidence-Based Complementary and Alternative Medicine, 1, 1753673. https://dx.doi.org/10.1155/2017/1753673

Pereira, V. V., Silva, R. R., Dos Santos, M., Dias, D. F., Moreira, M., \& Takahashi, J.A. (2015). Antioedematogenic activity, acetylcholinesterase inhibition and antimicrobial properties of Jacaranda oxyphylla. Natural Product Research, 30(17), 1974-1979. http://dx.doi.org/10.1080/14786419.2015.1095744

Pereira, E. J. P., Vale, J. P. C., Silva, P. T., Lima, J. R., Alves, D. R., Costa, P. S., Rodrigues, T. H. S., Menezes, J. E. S. A., Morais, S. M., Bandeira, P. N., Fontenelle, R. O. S., \& Santos, H. S. (2018). Circadian rhythm, and antimicrobial and anticholinesterase activities of essential oils from Vitex gardneriana. Natural Product Communications, 13(5), 635-638. https://doi.org/10.1177/1934578X1801300528

Rhee, I. K., Van der Meent, M., Ingkaninan, K., \& Verpoorte, R. (2001). Screening for acetylcholinesterase inhibitors from from Amararyllidaceae using silica gel thin-layer chromatography in combination with bioactivity staining. Journal of Chromatography A, 915(1-2), 217-223. https://doi.org/10.1016/S00219673(01)00624-0

Ribeiro, P. R. E., Carvalho Neto, M. F., Chagas, E. A., Chagas, P. C., Takahashi, J. A., Melo, A. C. G. R., Santos, R. C. S., \& Melo Filho, A. A. (2019). Acetylcolonesterase inhibitor potential in cinnamon seed oil (Cinnamomum zeylanicum Nees) Lauraceae in Roraima, Brazil. Chemical Engineering Transactions, 75(1), 361-366. https://doi.org/10.3303/CET1975061

Santos, F. O., Lima, H. G., Rosa, S. S. S., Mercês, N. B., Serra, T. M., Uzeda, R. S., Reis, I. M. A., Botura, M. B., Branco, A., \& Batatinha, M. J. M. (2018). In vitro acaricide and anticholinesterase activities of digitaria insularis (Poaceae) against Rhipicephalus (Boophilus) microplus. Veterinary Parasitology, 255, 102-106. https://doi.org/10.1016/j.vetpar.20 18.04.003

Santos, R. C. S., Melo Filho, A. A., Chagas, E. A., Takahashi, J. A., Ferraz, V. P., Fernandez, I M., Ribeiro, P. R. E., Melo, A. C. G. R., \& Holanda, L. C. (2015). Chemical composition, antimicrobial and anti-acetylcholinesterase activities of essential oil from Lantana camara (Verbenaceae) flowers. Journal of Medicinal Plants Research, 9(35), 922-928. http://dx.doi.org/10.5897/jmpr2015.5919

Santos, T. C., Gomes, T. M., Pinto, B. A. S., Camara, A. L., \& Paes, A. M. A. (2018). Naturally occurring acetylcholinesterase inhibitors and their potential use for Alzheimer's disease therapy. Frontiers in Pharmacology, 9, 1192. https://dx.doi.org/10.3389/fphar.2018.01192

Santos, W. P., Carvalho, A. C. S., Estevam, C. S., Santana, A. E. G., \& Marçal, R. M. (2012). In vitro and ex vivo anticholinesterase activities of Erythrina velutina leaf extracts. Pharmaceutical Biology, 50(7), 919-924. http://dx.doi.org/10.3109/13880209.2011.649429

Silva, A. A. S., Ferreira Júnior, J. M., Silva, M. G. V., \& Morais, S. M. (2014). Phytochemistry and biological activities little lemon (Zanthoxylum syncarpum Tull.). Revista Brasileira de Higiene e Sanidade Animal, 8(1), 37-53. http://dx.doi.org/10.5935/1981-2965.20140003

Silva, A. A. S., Alexandre, J. B., Vieira, L. G., Rodrigues, S. P., Falcão, M. J. C., \& Morais, S. M. M. (2015). Estudo fitoquímico e atividades leishmanicida, anticolinestarásica e antioxidante de extratos de Annona glabra L. (Araticum Panã). Revista de Ciências Farmacêuticas Básica e Aplicada, 36(2),189-194. https://rcfba.fcfar.unesp.br/index.php/ojs/article/view/43/42 
Research, Society and Development, v. 11, n. 1, e6211124262, 2022

(CC BY 4.0) | ISSN 2525-3409 | DOI: http://dx.doi.org/10.33448/rsd-v11i1.24262

Silva, J. K. R., Pinto, L. C., Burbano, R. M. R., Montenegro, R. C., Guimarães, E. F., Andrade, E. H. A., \& Maia, J. G. S. (2014). Essential oils of Amazon Piper species and their cytotoxic, antifungal, antioxidant and anti-cholinesterase activities. Industrial Crops And Products, 58(1), 55-60. http://dx.doi.org/10.1016/j.indcrop.2014.04.006

Silva, S. G., Costa, R. A., Oliveira, M. S., Cruz, J. N., Figueiredo, P. L. B., Brasil, D. S. B., Nascimento, L. D., Chaves Neto, A. M. J., Carvalho Junior, R. N., \& Andrade, E. H. A.(2019). Chemical profile of Lippia thymoides, evaluation of the acetylcholinesterase inhibitory activity of its essential oil, and molecular docking and molecular dynamics simulations, PLoS ONE, 14(3), e0213393. https://doi.org/10.1371/journal.pone.0213393.

Trevisan, M. T. S., Bezerra, M. Z. B., Santiago, G. M. P., Feitosa, C. M., Verpoorte, R. Braz Filho, R. (2006). Atividades larvicida e anticolinesterásica de plantas do gênero Kalanchoe. Química Nova, 29(3), 415-418. http://dx.doi.org/10.1590/s0100-40422006000300002.

Valli, M., Young, M. C. M., \& Bolzani, V. S. The invisible beauty of the biodiversity: the rubiaceae taxon. (2016). Revista Virtual de Química, 8(1), 296-310. https://doi.org/10.5935/1984-6835.20160020

Vinutha, B., Prashanth, D., Salma, K., Sreeja, S. L., Pratiti, D., Padmaja, R., Radhika, S., Amit, A., Venkateshwarlu, K., Deepak, M. (2007). Screening of selected Indian medicinal plants for acetylcholinesterase inhibitory activity. Journal of Ethnopharmacology, 109, 359-363. https://doi.org/10.1016/j.jep.2006.06.014

Yamaguchi, K. K. L., Alcântara, J. M., \& Veiga Junior, V. F. (2012). Investigação do potencial antioxidante e anticolinesterásico de 20 espécies da família Lauraceae. Acta Amazônia, 42(4), 541-546. https://doi.org/10.1590/S0044-59672012000400012 